\title{
Immune-Mediated Disease Flares or New-Onset Disease in 27 Subjects Following mRNA/DNA SARS-CoV-2 Vaccination
}

\author{
Abdulla Watad 1,2,3,4®D, Gabriele De Marco ${ }^{4}$, Hussein Mahajna ${ }^{2,5}$, Amit Druyan ${ }^{2,3}$, Mailam Eltity ${ }^{6}$, Nizar Hijazi ${ }^{7}$, \\ Amir Haddad 7,8, Muna Elias 7,8, Devy Zisman 7,8(-), Mohammad E. Naffaa 9 ${ }^{10}$, Michal Brodavka 2,3, Yael Cohen 2,3, \\ Arsalan Abu-Much ${ }^{10}$, Muhanad Abu Elhija ${ }^{7,8}$, Charlie Bridgewood ${ }^{4}$, Pnina Langevitz ${ }^{2,3}$, Joanna McLorinan ${ }^{11}$, \\ Nicola Luigi Bragazzi ${ }^{12,13, *(\mathbb{D})}$, Helena Marzo-Ortega ${ }^{4}\left(\mathbb{D}\right.$, Merav Lidar ${ }^{2,3}$, Cassandra Calabrese ${ }^{14}$, \\ Leonard Calabrese ${ }^{14}$, Edward Vital ${ }^{4}$, Yehuda Shoenfeld ${ }^{1,2}$, Howard Amital ${ }^{1,2,3,+}$ and Dennis McGonagle ${ }^{4, *,+}$
}

Citation: Watad, A.; De Marco, G.; Mahajna, H.; Druyan, A.; Eltity, M.; Hijazi, N.; Haddad, A.; Elias, M.; Zisman, D.; Naffaa, M.E.; et al. Immune-Mediated Disease Flares or New-Onset Disease in 27 Subjects Following mRNA/DNA SARS-CoV-2 Vaccination. Vaccines 2021, 9, 435. https://doi.org/10.3390/vaccines 9050435

Academic Editor:

Eduardo Gomez-Casado

Received: 14 March 2021

Accepted: 23 April 2021

Published: 29 April 2021

Publisher's Note: MDPI stays neutral with regard to jurisdictional claims in published maps and institutional affiliations.

Copyright: (c) 2021 by the authors. Licensee MDPI, Basel, Switzerland. This article is an open access article distributed under the terms and conditions of the Creative Commons Attribution (CC BY) license (https:// creativecommons.org/licenses/by/ $4.0 /)$
1 Department of Medicine 'B, Zabludowicz Center for Autoimmune Diseases, Sheba Medical Center, Tel-Hashomer 10457, Israel; watad.abdulla@gmail.com (A.W.); yehuda.shoenfeld@sheba.health.gov.il (Y.S.); Howard.Amital@sheba.health.gov.il (H.A.)

2 Sackler Faculty of Medicine, Tel-Aviv University, Tel-Aviv 69978, Israel; hmahajna@gmail.com (H.M.); Amit.Druyan@sheba.health.gov.il (A.D.); michal.brodavka@sheba.health.gov.il (M.B.); Yael.cohen2@sheba.health.gov.il (Y.C.); Pnina.Langevitz@sheba.health.gov.il (P.L.); Merav.Lidar@sheba.health.gov.il (M.L.)

3 Rheumatology Unit, Sheba Medical Center, Tel-Hashomer 10457, Israel

4 NIHR, Leeds Biomedical Research Centre, The Leeds Teaching Hospitals NHS Trust \& Leeds Institute of Rheumatic and Musculoskeletal Medicine, University of Leeds, Leeds LS9 7TF, UK; G.DeMarco@leeds.ac.uk (G.D.M.); C.D.Bridgewood@leeds.ac.uk (C.B.); h.marzo-ortega@leeds.ac.uk (H.M.-O.); E.M.J.Vital@leeds.ac.uk (E.V.)

5 Gastroenterology Department, Sheba Medical Center, Tel-Aviv 10457, Israel

6 Department of Neurology, Sheba Medical Center, Tel-Aviv 10457, Israel; mailameltity@gmail.com

7 Rheumatology Unit, Carmel Medical Center, Michal Street, Haifa 3436212, Israel; NizarHi@clalit.org.il (N.H.); haddadamir@yahoo.com (A.H.); nelias1@bezeqint.net (M.E.); devyzisman@gmail.com (D.Z.); Mhndhija100@gmail.com (M.A.E.)

8 Ruth and Bruce Rappaport Faculty of Medicine, Technion-Israel Institute of Technology, Haifa 3200003, Israel

9 Department of Rheumatology, Galilee Medical Center, Azrieli Faculty of Medicine, Bar-Ilan University, Safed 22100, Israel; MohammadN@gmc.gov.il

10 Leviev Heart Center, Sheba Medical Center, Tel Hashomer, Tel Aviv 10457, Israel; arsalanabumuch1@gmail.com

11 Department of Rheumatology, Mid Yorkshire Hospitals, West Yorkshire WF8 1PL, UK; joanna.mclorinan1@nhs.net

12 Centre for Disease Modelling, Department of Mathematics and Statistics, York University, Toronto, ON M3J 1P3, Canada

13 Fields-CQAM Laboratory of Mathematics for Public Health (MfPH), York University, Toronto, ON M3J 1P3, Canada

14 Cleveland Clinic Foundation, 9500 Euclid Avenue, Desk A50, Cleveland, OH 44195, USA; calabrc@ccf.org (C.C.); calabrl@ccf.org (L.C.)

* Correspondence: bragazzi@yorku.ca (N.L.B.); D.G.McGonagle@leeds.ac.uk (D.M.)

$+\quad$ These authors contributed equally to this study.

Abstract: Background: Infectious diseases and vaccines can occasionally cause new-onset or flare of immune-mediated diseases (IMDs). The adjuvanticity of the available SARS-CoV-2 vaccines is based on either TLR-7/8 or TLR-9 agonism, which is distinct from previous vaccines and is a common pathogenic mechanism in IMDs. Methods: We evaluated IMD flares or new disease onset within 28-days of SARS-CoV-2 vaccination at five large tertiary centres in countries with early vaccination adoption, three in Israel, one in UK, and one in USA. We assessed the pattern of disease expression in terms of autoimmune, autoinflammatory, or mixed disease phenotype and organ system affected. We also evaluated outcomes. Findings: 27 cases included 17 flares and 10 new onset IMDs. 23/27 received the BNT - 162b2 vaccine, $2 / 27$ the mRNA-1273 and 2/27 the ChAdOx 1 vaccines. The mean age was $54.4 \pm 19.2$ years and $55 \%$ of cases were female. Among the 27 cases, $21(78 \%)$ had at least one underlying autoimmune/rheumatic disease prior the vaccination. Among those patients with a flare or activation, four episodes occurred after receiving the second-dose and in one patient they occurred both after the first and the second-dose. In those patients with a new 
onset disease, two occurred after the second-dose and in one patient occurred both after the first (new onset) and second-dose (flare). For either dose, IMDs occurred on average 4 days later. Of the cases, $20 / 27$ (75\%) were mild to moderate in severity. Over $80 \%$ of cases had excellent resolution of inflammatory features, mostly with the use of corticosteroid therapy. Other immune-mediated conditions included idiopathic pericarditis $(n=2)$, neurosarcoidosis with small fiber neuropathy $(n=1)$, demyelination $(n=1)$, and myasthenia gravis $(n=2)$. In 22 cases $(81.5 \%)$, the insurgence of Adverse event following immunization (AEFI)/IMD could not be explained based on the drug received by the patient. In 23 cases (85.2\%), AEFI development could not be explained based on the underlying disease/co-morbidities. Only in one case (3.7\%), the timing window of the insurgence of the side effect was considered not compatible with the time from vaccine to flare. Interpretation: Despite the high population exposure in the regions served by these centers, IMDs flares or onset temporally-associated with SARS-CoV-2 vaccination appear rare. Most are moderate in severity and responsive to therapy although some severe flares occurred. Funding: none.

Keywords: vaccine safety; COVID-19; mRNA-based vaccine; adenoviral vector-based vaccine; immune-mediated diseases

\section{Introduction}

Since its initial outbreak in late December 2019, the "Severe Acute Respiratory Syndromerelated Coronavirus type 2" (SARS-CoV-2) infection has resulted in over 3.1 million deaths and has contributed to immeasurable additional medical and economic consequences due to the lockdown measures designed to control the virus spread and reduce disease mortality [1]. The strategy to end the "Coronavirus Disease 2019" (COVID-19) pandemic rests almost entirely on the few drugs available approved by regulatory bodies, such as the United States of America (US) Food and Drug Administration (FDA), like Remdesivir [2], and on vaccines, which, as a result, have been developed rapidly through global collaborations and unprecedented efforts $[3,4]$.

While vaccinations usually represent a safe and effective tool against infectious diseases, several critical steps of their development present distinct challenges. The US Centers for Disease Control and Prevention (CDC) have identified six stages: namely, the (i) exploratory, (ii) pre-clinical, (iii) clinical development, (iv) regulatory review and approval, (v) manufacturing, and (vi) quality control phases [5]. Consequently, achieving a safe, effective vaccine is a time- and resource-consuming process that ordinarily takes up to 10-15 years on average to be successfully completed. Conversely, in a rapidly evolving pandemic, such a protracted process may cost many more lives than it could save. Hence, due to the emergency situation related to the COVID-19 pandemic, several steps of the path to prevention have been fast-tracked. This has increased uncertainties about the longterm efficacy and safety of vaccines [6]. This abbreviated development process, therefore, warrants an increased scrutiny in post-marketing surveillance of biologically plausible side effects. Among these are immune-mediated phenomena.

To date, authorized COVID-19 vaccine products and those pending approval have shown good efficacy, excellent safety, and tolerability in randomized clinical trials (RCTs) [7-12]. Additionally, the first SARS-CoV-2 vaccine to the market-BNT162b2 (Pfizer/BioNTech) - has seen high utilization in several jurisdictions including the United Kingdom (UK), Israel and the USA, where vaccine roll-out commenced in early-to mid-December 2020. Although millions of individuals have been vaccinated, there have been so far few reports of vaccineassociated immune-mediated disease (IMD) development, with the only notable exception of rare, potentially immune-mediated autoimmune thrombosis [13].

The role of infectious agent derived antigens, including SARS-CoV-2 [14], as an IMD trigger, is well recognized with both natural infection or, less commonly, after vaccination. An array of innate and adaptive immune mechanisms, including molecular mimicry, may be responsible for these phenomena $[15,16]$. Adjuvants are a group of substances that drive 
innate immune system pattern recognition receptor (PRR) activation. They are commonly used in vaccines to boost immune reactivity towards target antigens [17,18]. A link between disorders that are characterized by innate and adaptive immune system dysregulation and exposure to an adjuvant such as aluminum, and squalene, has been reported [19-21]. These disorders, together with others, encompass a broad spectrum of reactions, which collectively have been referred to as the "vaccine adjuvant-related syndrome", term coined by Gherardi in 2003 [22] and, subsequently, as the "Autoimmune/Autoinflammatory Syndrome Induced by Adjuvants" (ASIA syndrome) [23]. However, these two syndromes remain highly controversial entities [24,25].

The adjuvanticity of the available SARS-CoV-2 vaccines is novel and, to a large degree, depends on the intrinsic adjuvanticity of vaccine messenger RNA (mRNA) or DNA which, respectively, stimulates the innate immunity through endosolic and cytoplasmic nucleic acid receptors such as Toll-Like Receptors (TLRs) 3, 7, 8, and 9, and components of the inflammasome, including Retinoic acid-Inducible Gene I (RIG-I) and Melanoma Differentiation-Associated Gene 5 (MDA5) [26,27].

Several IMDs, most notably autoimmune connective tissue diseases, may be linked to altered nucleic acid metabolism and processing, which have been shown to stimulate TLR-7 and TLR-9 experimentally and in humans [28,29]. However, vaccine development programs are not generally performed on subjects with IMDs where issues such as disease activity and immunotherapy may alter the course of disease. These diseases are too uncommon for such events to be detected in standard vaccine trial populations [30]. They affect about $3-7 \%$ of the general population with an estimated incidence of 80 per $10^{5}$ person-years [30]. Moreover, vaccine safety evaluation is generally considered as a secondary objective, with pivotal RCTs being underpowered to enable statistical analyses of endpoints of specific side effects, including IMDs [31]. Further, some adverse events could be hard to identify, especially those of vaccines produced utilizing new technologies [31].

During a pandemic, it is of paramount importance to provide real-time data concerning vaccine safety. Exchange of information, even though preliminary, can shed light on potential mechanisms underlying vaccine action [31] and generate hypotheses that can be further tested by ad hoc, large epidemiological surveys. Frontline physicians and public health-workers, who are the point of first contact in case of insurgence of an adverse event, can play a key role in this.

There are two major ways for identifying and reporting side effects following vaccination: namely, active, or passive surveillance. The former generally implies data mining of large electronic health record systems and is able to capture more adverse events than passive surveillance, even if most of them are usually mild. The latter system, being on a voluntary basis, is biased towards the reporting of more severe and earlier adverse events and can have multiple shortcomings, such as under-reporting of mild symptoms, unconfirmed diagnosis, and not always clear temporal links between vaccine administration and symptoms development. Even if the system generated by passive surveillance constitutes the backbone of the system exploited by active surveillance, only the latter can enable a rigorous analysis and comparison of epidemiological trends (i.e., incidence/prevalence rates) of adverse events following immunization, in terms of spatio-temporal trend and stratifying them according to the vaccination status [31].

Given the constantly evolving situation and the uncertainty about the still ongoing COVID-19 pandemic, it is crucial to collect data directly from frontline physicians [32].

Accordingly, we evaluated clinical networks in three countries at the vanguard of SARS-CoV-2 vaccination programs looking for evidence of new-onset or flare of immune disease temporally linked to vaccine administration, collecting data from collaborating physicians, utilizing reliable, confirmed diagnosis, and including also 1-month delayed reported symptoms. 


\section{Methods}

This study was reported according to the "CAse REports" (CARE) guidelines [33]. Verbal or written consent was obtained from all patients for the anonymized use of data. Data were obtained from three countries, Israel (Sheba Medical Centre in Tel Aviv, Carmel Hospital in Haifa, and Galilee Medical Centre in Nahariya), UK (The Leeds Teaching Hospitals NHS Trust) and USA (Cleveland Clinic). All patient events reported occurred in the study period December 2020 to February 2021.

We identified patients presenting with IMD, especially rheumatic diseases, including new presentations, new disease relapses or severe disease worsening that developed shortly after vaccination. Given that the most commonly recognized post infectious disease and post vaccination type of arthritis, termed reactive arthritis, may be diagnosed when onset is within one month of infection exposure, we took a cut-off of 28 days post vaccination as relevant.

According to the World Health Organization (WHO) guidelines [34], "Adverse Events Following Immunization" (AEFI) can be defined as any untoward, unfavorable, or unintended medical occurrence (ranging from indication, symptom, series of symptoms, disease, to abnormal laboratory findings) following vaccination, not necessarily exhibiting a consistent, causal relationship with the administration of the vaccine product. Based on the nature of the causal association, AEFIs can be classified into: (i) vaccine product-, (ii) vaccine quality defect-related reactions, (iii) immunization error or anxiety, and (iv) co-incidental event. Vaccine can act either as a factor or co-factor together with underlying predisposing conditions related to the biological or genetic make-up of the individual and the interaction of environmental variables. AEFIs are, as such, concrete examples of complex, multi-factorial events [35].

We evaluated the causality of the AEFI after COVID-19 vaccination based on the WHO guidelines, which propose a comprehensive four-step analytical and algorithmic diagramming process. Even though the WHO instrument has been criticized [35], currently, there exist no valid and reliable alternatives [36]. All possible "other causes" that could explain the insurgence of the AEFI, excluding the etiopathological role of the vaccine, were initially considered. Notably, all patients were well controlled and reported general well-being with pharmacological therapy prior to vaccination, with the exception of a case in which the administration of rituximab was delayed for immunization purpose. After validating IMD diagnosis, and excluding non-vaccination related causalities, biological plausibility and temporal compatibility between the immunization and the occurrence of the AEFI were assessed (Figure 1). To ensure a reliable assessment of AEFIs, a multi-disciplinary evaluation was performed, involving different specialists, ranging from immunologists, rheumatologists, internal medicine doctors and epidemiologists, as recommended by the WHO guidelines. Since these guidelines and checklists concern general AEFIs and not particular classes of adverse events after immunization, similarly to the approach outlined in [36], we have adapted them to the specific case of IMD-like side effects (Figure 1).

Moreover, based on the type of IMD, AEFIs were categorized according to the immunologic disease continuum for the classification of vaccine reactions proposed by Koenig et al. [37].

This scheme includes the following categories including "classical adaptive immunemeditated diseases" (mainly involving B- and T-cells and primary lymphoid organs), "innate immune-mediated diseases" (affecting cells of the innate immune system) and "intermediate diseases" (including major histocompatibility complex (MHC) class I-related disease), "non-immunoglobulin E (IgE)- and IgE-related hypersensitivity" and "innate immune driven" or autoinflammatory diseases as earlier proposed [38].

Descriptive statistics were used to summarize the age, sex, history of IMD, the average of onset of symptoms, severity, therapeutics administered, and key clinical and laboratory findings. 


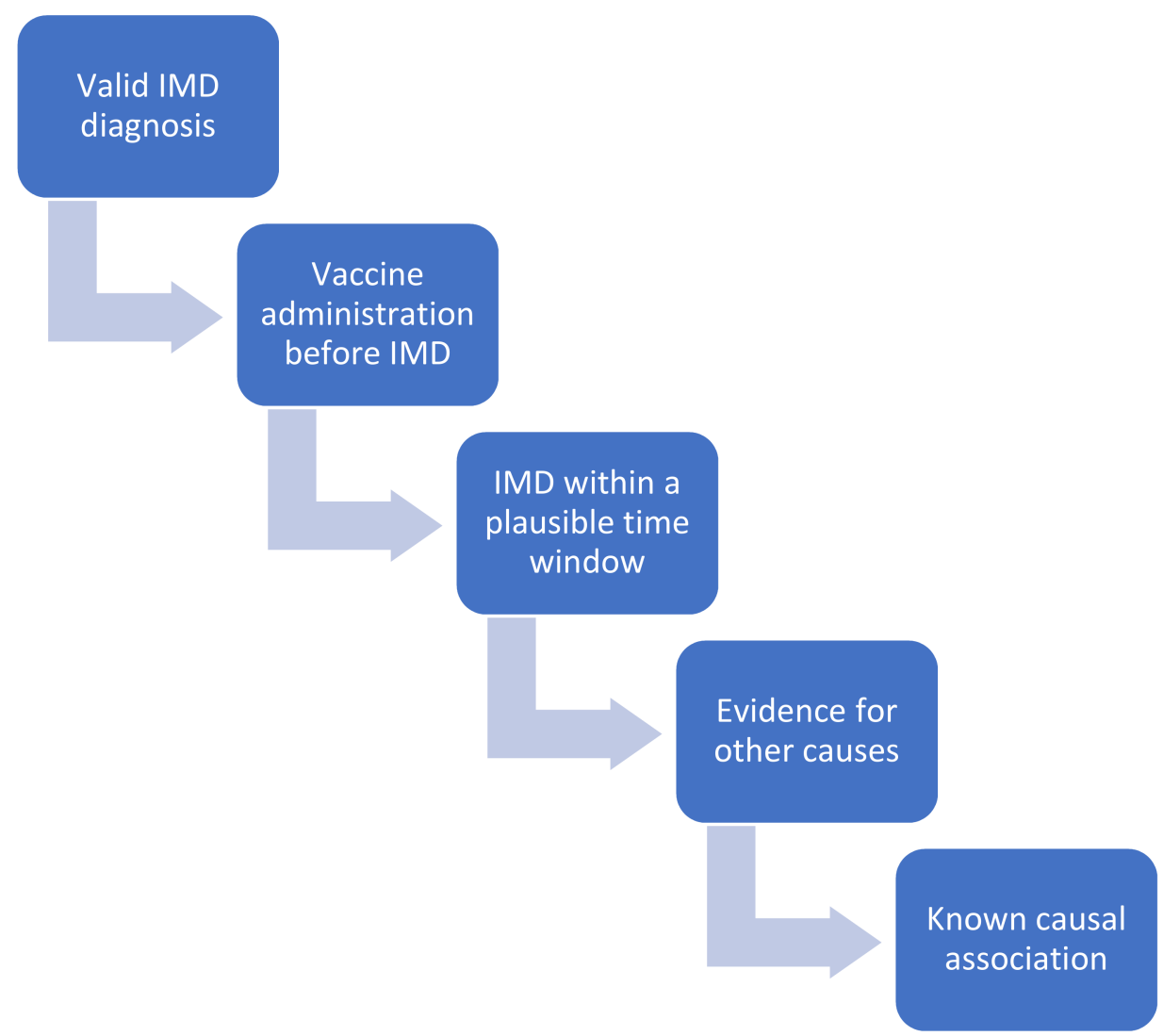

Figure 1. Causality assessment of “Adverse Events Following Immunization" (AEFIs) after “Coronavirus Disease 2019" (COVID-19) vaccination based on the World Health Organization (WHO) guidelines, which propose a comprehensive four-step analytical and algorithmic diagramming process (namely, evaluation and assessment of (i) the temporal association between vaccine administration and AEFI/immune-mediated disease (IMD); (ii) a plausible time window between vaccine administration and AEFI/IMD; (iii) other causes, such as underlying co-morbidities or drugs taken by the patient, which could explain the insurgence of AEFI/IMD; and, (iv) strength of the causal association, based on what is currently known from the literature).

\section{Results}

We identified 27 cases of IMDs (15 females, 55.6\%; 12 males, $44.4 \%$; mean age $54.44 \pm 19.20$ years). 21 had an autoimmune/rheumatic background. This could be further subcategorized as autoimmune ( $n=11,52.4 \%)$, autoinflammatory $(n=4,19.0 \%)$ and mixed pattern $(n=6,28.6 \%)$ disorders before COVID-19 vaccination. In six patients $(22.2 \%)$, there was no autoimmune/rheumatic background and patients presented new-onset rheumatic and musculoskeletal (RMD) and non-RMD disorders (Table 1).

Other factors such as alternative infectious triggers, active COVID-19 infection around vaccination, joint trauma or major surgery as triggering events were not found, although patients were not systematically tested for SARS-CoV-2 infection unless there were typical symptoms.

Twenty-three (85.2\%), two (7.4\%), and two (7.4\%) received the BNT-162b2, mRNA1273 and ChAdOx1 vaccines, respectively. Twenty cases $(74.1 \%)$ were reported in Israel, five $(18.5 \%)$ in the UK, and two $(7.4 \%)$ in the USA. 
Table 1. The study population basic characteristics. Abbreviations: NSAIDs: nonsteroidal antiinflammatory drugs.

\begin{tabular}{|c|c|}
\hline Parameter & Value \\
\hline \multicolumn{2}{|l|}{ Sex } \\
\hline Female & $15(55.6 \%)$ \\
\hline Male & $12(44.4 \%)$ \\
\hline Age (mean \pm standard deviation; median) & $54.44 \pm 19.20 ; 55$ \\
\hline \multicolumn{2}{|l|}{ Pre-vaccine autoimmune/rheumatic history } \\
\hline None & $6(22.2 \%)$ \\
\hline Rheumatoid Arthritis & $6(22.2 \%)$ \\
\hline Behçet's disease & $4(14.8 \%)$ \\
\hline Systemic lupus erythematosus (SLE)/SLE-like & $3(11.1 \%)$ \\
\hline Pericarditis & $2(7.4 \%)$ \\
\hline Others & $6(22.2 \%)$ \\
\hline \multicolumn{2}{|l|}{ Vaccine } \\
\hline BNT-162b2 & $23(85.2 \%)$ \\
\hline mRNA-1273 & $2(7.4 \%)$ \\
\hline ChAdOx1 & $2(7.4 \%)$ \\
\hline \multicolumn{2}{|l|}{ 2nd Vaccine } \\
\hline Administered & $12(44.4 \%)$ \\
\hline Not administered yet & $15(55.6 \%)$ \\
\hline \multicolumn{2}{|l|}{ Days from vaccine to flare/new-onset } \\
\hline After 1st dose & $21 / 27(77.8 \%) ;$ median 4 days [1-25 days] \\
\hline After 2nd dose & $8 / 12(66.7 \%) ;$ median 4 days [1-7 days] \\
\hline Flare & $17(63.0 \%)$ \\
\hline New-onset & $10(37.0 \%)$ \\
\hline \multicolumn{2}{|l|}{ Severity } \\
\hline Mild & $6(22.2 \%)$ \\
\hline Moderate & $14(51.9 \%)$ \\
\hline Severe & $7(25.9 \%)$ \\
\hline \multicolumn{2}{|l|}{ Therapy } \\
\hline Glucocorticoids & $21(77.8 \%)$ \\
\hline Colchicine & $4(14.8 \%)$ \\
\hline NSAIDs & $3(11.1 \%)$ \\
\hline Plasma exchange & $2(7.4 \%)$ \\
\hline Hydroxychloroquine & $1(3.7 \%)$ \\
\hline Rituximab & $1(3.7 \%)$ \\
\hline Local steroids & $1(3.7 \%)$ \\
\hline Pyridostigmine & $1(3.7 \%)$ \\
\hline Combination of drugs & $7(25.9 \%)$ \\
\hline No additional treatment & $1(3.7 \%)$ \\
\hline \multicolumn{2}{|l|}{ Response } \\
\hline Rapid response & $21(77.8 \%)$ \\
\hline Spontaneous resolution & $2(7.4 \%)$ \\
\hline Slow response & $2(7.4 \%)$ \\
\hline Intubation & $1(3.7 \%)$ \\
\hline
\end{tabular}

The average time between vaccination and new-onset or flare of symptoms was 4 days (median of 4 days [1-25 days] in those who developed an IMD after the first dose and a median of 4 days [1-7 days] in those after the second dose) with most cases occurring after the first inoculation (77.8\%). Twelve cases flared after the first vaccine dose, one case flared after both the first and the second vaccine dose, and four cases flared only after the second vaccine dose. Fifteen cases (55.6\%) did not receive the second vaccination.

Although we did not formally collect data on well-known vaccine adverse reactions such as fever, headaches, myalgia, and arm pain following vaccination, these features did 
not appear to be severe from review of medical records. Most IMDs were mild-to-moderate in terms of severity $(n=20,74.1 \%)$.

In 22 cases $(81.5 \%)$, the development of AEFI/IMD could not be explained based on the drug received by the patient. In 23 cases (85.2\%), AEFI development could not be explained based on the underlying disease/co-morbidities. Only in one case (3.7\%), the timing window of the insurgence of the side effect was considered not compatible with the time from vaccine to flare (Table 1).

\subsection{RMD Cases}

The individual cases are summarized in Table 2.

Overall, 20/27 cases had RMD disease. Among those, 11 patients had arthritis, 9 flares, and 2 of new-onset, and in 8/11 patients, arthritis was the only presentation without extra-articular features, 7 cases of arthritis were seronegative inflammatory arthritis and 3 were anti-citrullinated protein antibody positive $(\mathrm{ACPA}+)$, rheumatoid factor positive $(\mathrm{RF}+)$ autoimmune and 1 case of mixed pattern nature (Figure 1 ). One case of a flare in gout was noted with flare in this arthropathy being well reported with native SARS-CoV-2 infection during the first wave of the pandemic [39].

Four cases of Behcet's disease (BD) flare were noted in Israel with all of these having oral ulceration flares with one experiencing associated arthritis. None of the BD cases experienced major internal organ flares or immunothrombotic vascular episodes with disease limited to cutaneous and articular flares (Figure 2).

With respect to autoimmune connective tissue diseases, 3 cases of systemic lupus erythematosus (SLE) (2 flares and one case with predominant discoid SLE clinical features that converted to confirmed pleuropericarditis) were noted. A single case of known dermatomyositis experienced a flare in cutaneous disease but not myositis following vaccination (Table 2) (Figure 3B).

Three vasculitis cases, all new-onset were reported including a Henoch-Schönlein Purpura (HSP) pattern, and 2 cases of chilblain lesions (Figure 2).

One new case of polymyalgia rheumatica (PMR) was reported and 1 case of remitting seronegative symmetrical synovitis with pitting edema (RS3PE) (new-onset) was documented in a subject with confirmed PMR that was treated 15 years earlier. No cases of Giant cell arteritis (GCA) were noted.

\subsection{Non-RMD}

The individual cases are summarized in Table 2. In our study, 7 non-RMD cases were reported. Among these, 4 autoimmune neurological disorders were found. Two new-onset myasthenia gravis cases occurred, both after the second dose of BNT162b2 vaccine, one being severe. A single new-onset case of multiple sclerosis (MS) was reported after a week after the first dose of BNT162b2 vaccine, which was clinically moderate and responded well to therapy (Figure 4, in particular Figure 4C). One moderate flare case of neurosarcoidosis and small fiber neuropathy that resolved spontaneously was also reported.

We observed two cases of pericarditis that we considered to be more autoinflammatory than autoimmune (Figure 4, in particular Figure 4A,B). Notably, one case was particularly suggestive of vaccine-induced inflammation as with each vaccination, pericarditis occurred 4 days after the first dose and a flare 4 days after the second dose. Both episodes were treated with nonsteroidal anti-inflammatory drugs (NSAIDs) and colchicine and responded well.

An additional case of pericarditis flare was noted in an ACPA+ RF+ RA case but there was no flare in joint disease. Miscellaneous diagnosis cases included a case of ulcerative colitis (UC) and severe associated hypereosinophilic syndrome that flared. 


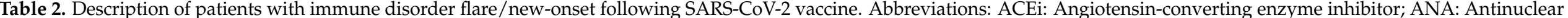

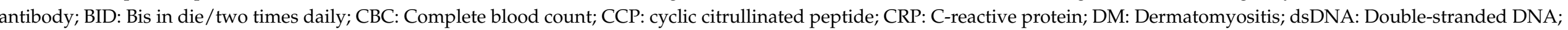

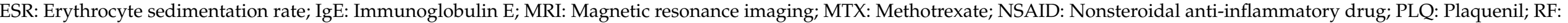

Rheumatoid factor; RA: Rheumatoid arthritis; RS3PE: Remitting seronegative symmetrical synovitis with pitting oedema; SLE: Systemic lupus erythematosus; UC: Ulcerative colitis.

\begin{tabular}{|c|c|c|c|c|c|c|c|c|c|c|c|c|c|c|c|c|}
\hline ID & Sex & Age & $\begin{array}{l}\text { Pre-Vaccine } \\
\text { History of } \\
\text { Immune } \\
\text { Mediated } \\
\text { Disease + } \\
\text { Medication }\end{array}$ & $\begin{array}{l}\text { Date 1st } \\
\text { Vaccine } \\
\text { Dose }\end{array}$ & $\begin{array}{l}\text { Date } \\
\text { 2nd } \\
\text { Vaccine } \\
\text { Dose }\end{array}$ & 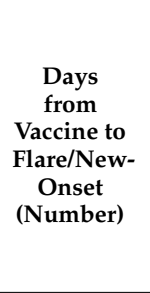 & $\begin{array}{c}\text { Immune } \\
\text { Mediated } \\
\text { Disease } \\
\text { Complication }\end{array}$ & $\begin{array}{l}\text { Flare } \\
\text { (F)/New- } \\
\text { Onset } \\
(\mathrm{N})\end{array}$ & Severity & $\begin{array}{l}\text { Relevant Lab } \\
\text { Tests }\end{array}$ & Therapy & $\begin{array}{l}\text { Comments on } \\
\text { Therapy and } \\
\text { Response }\end{array}$ & Country & $\begin{array}{c}\text { Can the } \\
\text { reaction } \\
\text { be } \\
\text { Explained } \\
\text { Based on } \\
\text { Drug } \\
\text { Received } \\
\text { by the } \\
\text { Patient? } \\
\text { (Yes/No) }\end{array}$ & $\begin{array}{c}\text { Can the } \\
\text { Reaction be } \\
\text { Explained } \\
\text { Based on the } \\
\text { Underlying } \\
\text { Co- } \\
\text { Morbidity } \\
\text { of the } \\
\text { Patient? } \\
\text { (Yes/No) }\end{array}$ & $\begin{array}{c}\text { Do you Feel } \\
\text { the Timing } \\
\text { of the } \\
\text { Side-Effect } \\
\text { Compatible } \\
\text { with the } \\
\text { Time from } \\
\text { Vaccine to } \\
\text { Flare? } \\
\text { (Yes/No) }\end{array}$ \\
\hline 1 & $\mathrm{~F}$ & 83 & $\begin{array}{l}\text { Polymyalgia } \\
\text { rheumatica since } \\
2005 \text { (clinical } \\
\text { diagnosis) } \\
\text { Hypothyroidism }\end{array}$ & $\begin{array}{l}\text { January } \\
2021 \\
\text { BNT162b2 } \\
\text { vaccine }\end{array}$ & $\begin{array}{l}\text { Not } \\
\text { given } \\
\text { yet }\end{array}$ & $\begin{array}{c}7 \text { days } \\
\text { (after dose } \\
\text { 1) }\end{array}$ & $\begin{array}{c}\text { Severe } \\
\text { Seronegative } \\
\text { Polyarthritis with } \\
\text { RSSPE pattern } \\
\text { (clinical } \\
\text { assessment) }\end{array}$ & $\mathrm{N}$ & 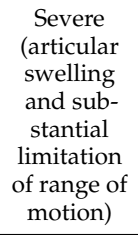 & $\begin{array}{c}\text { CRP } 74 \mathrm{mg} / \mathrm{L} ; \\
\text { ANA screening } \\
\text { (including } \\
\text { anti-dsDNA) } \\
\text { negative; } \\
\text { Anti-CCP } \\
\text { negative; } \\
\text { RF negative }\end{array}$ & $\begin{array}{l}\text { Prednisolone } \\
15 \mathrm{mgs} \text { day }\end{array}$ & $\begin{array}{l}\text { Rapid response } \\
\text { to therapy with } \\
\text { quick symptom } \\
\text { improvement }\end{array}$ & UK & Yes & No & Yes \\
\hline 2 & $\mathrm{~F}$ & 42 & $\begin{array}{c}\text { Transient } \\
\text { synovitis (one } \\
\text { episode in 2015, } \\
\text { clinical } \\
\text { diagnosis) }\end{array}$ & $\begin{array}{c}\text { January } \\
2021 \\
\text { BNT162b2 } \\
\text { vaccine }\end{array}$ & $\begin{array}{l}\text { Not } \\
\text { given }\end{array}$ & $\begin{array}{c}4 \text { days } \\
\text { (after dose } \\
\text { 1) }\end{array}$ & $\begin{array}{c}\text { Migratory } \\
\text { arthritis small } \\
\text { joints with } \\
\text { erythema and } \\
\text { hemorrhagic rash } \\
\text { on toes (clinical } \\
\text { assessment) }\end{array}$ & F & $\begin{array}{c}\text { Moderate } \\
\text { (articular } \\
\text { swelling } \\
\text { and } \\
\text { moderate } \\
\text { limitation } \\
\text { of range of } \\
\text { motion) }\end{array}$ & $\begin{array}{c}\mathrm{CRP}<4 \mathrm{mg} / \mathrm{L} \\
\text { ANA screening } \\
\text { (including } \\
\text { anti-dsDNA) } \\
\text { negative; } \\
\text { RF negative }\end{array}$ & $\begin{array}{l}\text { Prednisolone } \\
10 \text { mgs day }\end{array}$ & $\begin{array}{l}\text { Rapid response } \\
\text { to therapy with } \\
\text { low grade } \\
\text { swelling of } 4 \\
\text { joints without } \\
\text { tenderness after } 1 \\
\text { week }\end{array}$ & UK & Yes & No & Yes \\
\hline 3 & M & 43 & $\begin{array}{l}\text { Neurosarcoidos } \\
\text { is and small fiber } \\
\text { neuropathy, } \\
\text { diagnosed } 3 \\
\text { years prior to } \\
\text { vaccination. } \\
\text { treated with } \\
\text { infliximab for the } \\
\text { last } 2.5 \text { years and } \\
\text { asymptomatic } \\
\text { under treatment }\end{array}$ & $\begin{array}{l}\text { February } \\
2021 \\
\text { BNT162b2 } \\
\text { vaccine }\end{array}$ & $\begin{array}{l}\text { February } \\
2021\end{array}$ & $\begin{array}{c}3 \text { (after } \\
\text { first dose) }\end{array}$ & $\begin{array}{l}\text { Flare in } \\
\text { neurosarcoidosis } \\
\text { and neuropathy }\end{array}$ & $\mathrm{F}$ & Moderate & $\begin{array}{l}\text { MRI of the } \\
\text { spine did not } \\
\text { show relapse } \\
\text { of myelitis }\end{array}$ & $\begin{array}{c}\text { No } \\
\text { additional } \\
\text { treatment }\end{array}$ & $\begin{array}{c}\text { Flare } \\
\text { spontaneously } \\
\text { improved after } 2 \\
\text { weeks, the } \\
\text { patient received } \\
\text { 2nd dose of } \\
\text { vaccine with no } \\
\text { additional side } \\
\text { effects }\end{array}$ & Israel & No & Yes & Yes \\
\hline 4 & M & 38 & $\begin{array}{l}\text { Hypertension; } \\
\text { dyslipidemia, } \\
\text { and hypertrophic } \\
\text { cardiomyopathy, } \\
\text { treated with } \\
\text { beta-blockers, } \\
\text { ACEi, and } \\
\text { atorvastatin }\end{array}$ & $\begin{array}{c}\text { January } \\
2021 \\
\text { BNT162b2 } \\
\text { vaccine }\end{array}$ & $\begin{array}{l}\text { January } \\
2021 \\
\text { BNT162b2 } \\
\text { vaccine }\end{array}$ & $\begin{array}{l}\text { Onset (4 } \\
\text { days after } \\
\text { 1st dose) } \\
\text { and flare } \\
\text { (4 days } \\
\text { after 2nd } \\
\text { dose })\end{array}$ & Pericarditis & $\mathrm{N}$ & Moderate & $\begin{array}{l}\text { CRP 45, ESR } 60 . \\
\text { Negative for } \\
\text { ANA }\end{array}$ & $\begin{array}{l}\text { Treated with } \\
\text { NSAIDs and } \\
\text { colchicine. } \\
\text { (1st event) } \\
\text { and } 20 \mathrm{mg} \\
\text { prednisone } \\
\text { (2nd event) }\end{array}$ & $\begin{array}{l}\text { Rapid response } \\
\text { to therapy with } \\
\text { quick symptom } \\
\text { improvement }\end{array}$ & Israel & No & No & Yes \\
\hline
\end{tabular}


Table 2. Cont.

\begin{tabular}{|c|c|c|c|c|c|c|c|c|c|c|c|c|c|c|c|c|}
\hline ID & Sex & Age & $\begin{array}{l}\text { Pre-Vaccine } \\
\text { History of } \\
\text { Immune } \\
\text { Mediated } \\
\text { Disease + } \\
\text { Medication }\end{array}$ & $\begin{array}{l}\text { Date 1st } \\
\text { Vaccine } \\
\text { Dose }\end{array}$ & $\begin{array}{l}\text { Date } \\
\text { 2nd } \\
\text { Vaccine } \\
\text { Dose }\end{array}$ & $\begin{array}{l}\text { Days } \\
\text { from } \\
\text { Vaccine to } \\
\text { Flare/New- } \\
\text { Onset } \\
\text { (Number) }\end{array}$ & $\begin{array}{l}\text { Immune } \\
\text { Mediated } \\
\text { Disease } \\
\text { Complication }\end{array}$ & $\begin{array}{l}\text { Flare } \\
\text { (F)/New- } \\
\text { Onset } \\
\text { (N) }\end{array}$ & Severity & $\begin{array}{l}\text { Relevant Lab } \\
\text { Tests }\end{array}$ & Therapy & $\begin{array}{l}\text { Comments on } \\
\text { Therapy and } \\
\text { Response }\end{array}$ & Country & $\begin{array}{c}\text { Can the } \\
\text { reaction } \\
\text { be } \\
\text { Explained } \\
\text { Based on } \\
\text { Drug } \\
\text { Received } \\
\text { by the } \\
\text { Patient? } \\
\text { (Yes/No) }\end{array}$ & $\begin{array}{c}\text { Can the } \\
\text { Reaction be } \\
\text { Explained } \\
\text { Based on the } \\
\text { Underlying } \\
\text { Co- } \\
\text { Morbidity } \\
\text { of the } \\
\text { Patient? } \\
\text { (Yes/No) }\end{array}$ & $\begin{array}{c}\text { Do you Feel } \\
\text { the Timing } \\
\text { of the } \\
\text { Side-Effect } \\
\text { Compatible } \\
\text { with the } \\
\text { Time from } \\
\text { Vaccine to } \\
\text { Flare? } \\
\text { (Yes/No) }\end{array}$ \\
\hline 5 & M & 28 & $\begin{array}{c}\text { Ulcerative Colitis } \\
\text { (since } 10 \text { years) + } \\
\text { hypere- } \\
\text { osinophilic } \\
\text { syndrome (since } \\
5 \text { years) } \\
\text { (Vedoliziumab } \\
300 \text { mg every } 8 \\
\text { week and } \\
\text { cyclosporin } 150 \\
\text { mg BID) well } \\
\text { controlled for a } \\
\text { year }\end{array}$ & $\begin{array}{l}\text { January } \\
2021 \\
\text { BNT162b2 } \\
\text { vaccine }\end{array}$ & $\begin{array}{l}\text { Not } \\
\text { given }\end{array}$ & $\begin{array}{l}4 \text { days } \\
\text { after 1st } \\
\text { dose }\end{array}$ & $\begin{array}{l}\text { Flare of hypere- } \\
\text { osinophilic } \\
\text { syndrome and } \\
\text { UC with } \\
\text { vesicular skin } \\
\text { rash, oral } \\
\text { aphthosis and } \\
\text { hemorrhagic } \\
\text { diarrhea }\end{array}$ & $\mathrm{F}$ & Severe & $\begin{array}{l}\text { IgE } 41400,9000 \\
\text { eosinophils, } \\
\text { CRP } 35\end{array}$ & $\begin{array}{l}1 \text { gr of } \\
\text { sulomedrol } \\
\text { daily for } 3 \\
\text { days, then } \\
\text { prednisone } \\
60 \mathrm{mg} / \text { day } \\
\text { tapering } \\
\text { dose } \\
\text { (decrease } 20 \\
\text { mg every } 2 \\
\text { weeks) }\end{array}$ & $\begin{array}{c}\text { Slow response, } \\
\text { eosinophils of } \\
1200 \text { after } 2 \\
\text { weeks of therapy, } \\
\text { significant and } \\
\text { partial } \\
\text { improvement in } \\
\text { diarrhea and skin } \\
\text { rash, respectively. }\end{array}$ & Israel & No & Yes & Yes \\
\hline 6 & M & 70 & $\begin{array}{l}\text { No medical } \\
\text { history }\end{array}$ & $\begin{array}{l}\text { December } \\
2020 \\
\text { BNT162b2 } \\
\text { vaccine }\end{array}$ & $\begin{array}{l}\text { Not } \\
\text { given }\end{array}$ & $\begin{array}{l}3 \text { days } \\
\text { after 1st } \\
\text { dose }\end{array}$ & $\begin{array}{l}\text { Polymyalgia } \\
\text { rheumatica; } \\
\text { severe stiffness } \\
\text { and pain in } \\
\text { shoulders and } \\
\text { hips, fever and } \\
\text { fatigue }\end{array}$ & $\mathrm{N}$ & Severe & $\begin{array}{c}\text { CRP 175, ESR } \\
90 \\
\text { Anti-CCP, RF } \\
\text { and ANA } \\
\text { negative }\end{array}$ & $\begin{array}{l}\text { Prednisone } \\
\text { dose } 40 \mathrm{mg} \\
\text { once daily }\end{array}$ & $\begin{array}{l}\text { Rapid response } \\
\text { to therapy with } \\
\text { quick symptom } \\
\text { improvement }\end{array}$ & Israel & No & No & Yes \\
\hline 7 & $\mathrm{~F}$ & 45 & $\begin{array}{l}\text { Hypothyroidism } \\
\text { controlled with } \\
\text { Eltroxin } 50 \text { mcg } \\
\text { daily }\end{array}$ & $\begin{array}{l}\text { December } \\
2020 \\
\text { BNT162b2 } \\
\text { vaccine }\end{array}$ & $\begin{array}{c}\text { Not } \\
\text { given }\end{array}$ & $\begin{array}{l}7 \text { after the } \\
1 \text { st dose }\end{array}$ & $\begin{array}{l}\text { Multiple sclerosis } \\
\text { with left leg } \\
\text { weakness } \\
\text { disequilibrium, } \\
\text { and lower limbs } \\
\text { distal numbness }\end{array}$ & $\mathrm{N}$ & Moderate & $\begin{array}{c}\text { Normal CBC } \\
\text { and } \\
\text { biochemistry } \\
\text { Multiple Peri- } \\
\text { ventricular } \\
\text { white matter } \\
\text { changes on } \\
\text { MRI. } \\
\text { CSF- } \\
\text { oligoclonal } \\
\text { bands. }\end{array}$ & $\begin{array}{c}1 \mathrm{~g} \text { of } \\
\text { sulomedrol } \\
\text { daily for } 5 \\
\text { days, then } \\
\text { prednisone } \\
60 \text { mg daily } \\
\text { with } \\
\text { tapering } \\
\text { down dose }\end{array}$ & $\begin{array}{l}\text { Rapid response } \\
\text { to therapy with } \\
\text { quick symptom } \\
\text { improvement }\end{array}$ & Israel & No & No & No \\
\hline
\end{tabular}


Table 2. Cont.

\begin{tabular}{|c|c|c|c|c|c|c|c|c|c|c|c|c|c|c|c|c|}
\hline ID & Sex & Age & $\begin{array}{l}\text { Pre-Vaccine } \\
\text { History of } \\
\text { Immune } \\
\text { Mediated } \\
\text { Disease + } \\
\text { Medication }\end{array}$ & $\begin{array}{l}\text { Date 1st } \\
\text { Vaccine } \\
\text { Dose }\end{array}$ & $\begin{array}{l}\text { Date } \\
\text { 2nd } \\
\text { Vaccine } \\
\text { Dose }\end{array}$ & $\begin{array}{l}\text { Days } \\
\text { from } \\
\text { Vaccine to } \\
\text { Flare/New- } \\
\text { Onset } \\
\text { (Number) }\end{array}$ & $\begin{array}{c}\text { Immune } \\
\text { Mediated } \\
\text { Disease } \\
\text { Complication }\end{array}$ & $\begin{array}{l}\text { Flare } \\
\text { (F)/New- } \\
\text { Onset } \\
\text { (N) }\end{array}$ & Severity & $\begin{array}{l}\text { Relevant Lab } \\
\text { Tests }\end{array}$ & Therapy & $\begin{array}{l}\text { Comments on } \\
\text { Therapy and } \\
\text { Response }\end{array}$ & Country & $\begin{array}{c}\text { Can the } \\
\text { reaction } \\
\text { be } \\
\text { Explained } \\
\text { Based on } \\
\text { Drug } \\
\text { Received } \\
\text { by the } \\
\text { Patient? } \\
\text { (Yes/No) }\end{array}$ & $\begin{array}{c}\text { Can the } \\
\text { Reaction be } \\
\text { Explained } \\
\text { Based on the } \\
\text { Underlying } \\
\text { Co- } \\
\text { Morbidity } \\
\text { of the } \\
\text { Patient? } \\
\text { (Yes/No) }\end{array}$ & $\begin{array}{c}\text { Do you Feel } \\
\text { the Timing } \\
\text { of the } \\
\text { Side-Effect } \\
\text { Compatible } \\
\text { with the } \\
\text { Time from } \\
\text { Vaccine to } \\
\text { Flare? } \\
\text { (Yes/No) }\end{array}$ \\
\hline 8 & $\mathrm{~F}$ & 66 & $\begin{array}{c}\text { Idiopathic } \\
\text { pericarditis, } \\
\text { anemia } \\
\text { normocytic } \\
\text { normochromic, } \\
\text { left } \\
\text { femoro-popliteal } \\
\text { DVT treated with } \\
\text { rivaroxaban } 20 \\
\text { mg } 12.2020, \\
\text { spontaneous } \\
\text { abortion of 1st } \\
\text { trimester } \\
\text { Idiopathic } \\
\text { pericarditis }\end{array}$ & $\begin{array}{l}\text { January } \\
2021 \\
\text { BNT162b2 } \\
\text { vaccine }\end{array}$ & $\begin{array}{l}\text { Not } \\
\text { given }\end{array}$ & $\begin{array}{l}2 \text { days } \\
\text { after the } \\
1 \text { st dose }\end{array}$ & $\begin{array}{l}\text { Pericarditis with } \\
\text { fever, typical } \\
\text { pleuritic chest } \\
\text { pain, elevated } \\
\text { inflammation } \\
\text { markers } \\
\text { No RA articular } \\
\text { manifestation. }\end{array}$ & $\mathrm{F}$ & $\begin{array}{c}\text { Mild } \\
\text { (moderate } \\
\text { chest pain, } \\
\text { no } \\
\text { symptoms } \\
\text { of heart } \\
\text { failure, no } \\
\text { limitation } \\
\text { of } \\
\text { function) }\end{array}$ & $\begin{array}{l}\text { Positive ACPA } \\
\text { 5.4, RF 44, CRP } \\
\text { 40, ESR } 70\end{array}$ & $\begin{array}{c}\text { Continued } \\
\text { Prednisone } \\
15 \mathrm{mg} \\
\text { prednisone. } \\
\text { On } 19 \\
\text { January 2021, } \\
\text { dose was } \\
\text { increased to } \\
30 \mathrm{mg} \text { and } 1 \\
\text { mg of } \\
\text { colchicine. } \\
\text { by physician. } \\
15 \mathrm{mg} \text { of } \\
\text { Methotrex- } \\
\text { ate was } \\
\text { started } \\
\text { together } \\
\text { with } 5 \mathrm{mg} \text { of } \\
\text { folic acid }\end{array}$ & $\begin{array}{l}\text { Rapid response } \\
\text { to therapy with } \\
\text { quick symptom } \\
\text { improvement. }\end{array}$ & Israel & No & No & Yes \\
\hline 9 & $\mathrm{~F}$ & 36 & $\begin{array}{l}\text { Psoriasis since } \\
\text { childhood, mild }\end{array}$ & $\begin{array}{l}\text { December } \\
2020 \\
\text { MRNA- } \\
1273\end{array}$ & $\begin{array}{l}\text { January } \\
2021\end{array}$ & $\begin{array}{l}10 \text { days } \\
\text { after 1st } \\
\text { dose }\end{array}$ & $\begin{array}{l}\text { Dactylitis of RT } \\
\text { 3rd finger } \\
\text { Onset of R 2nd } \\
\text { and 3rd finger } \\
\text { joint pain, } \\
\text { stiffness and } \\
\text { tightness. Also } \\
\text { developed } \\
\text { painful } \\
\text { erythematous } \\
\text { macules over } \\
\text { palmar surface of } \\
\text { several fingers on } \\
\text { both hands } \\
\\
\text { Pain chilblains } \\
\text { like lesions on } \\
\text { fingers (painful) }\end{array}$ & $\mathrm{N}$ & Mild & $\begin{array}{c}\text { Normal CBC, } \\
\text { chemistry, ESR } \\
\text { and CRP. Mus- } \\
\text { culoskeletal US } \\
\text { without } \\
\text { synovitis or } \\
\text { tenosynovitis } \\
\text { (done } 1 / 27 \text {, } \\
\text { symptoms had } \\
\text { nearly } \\
\text { resolved) }\end{array}$ & $\begin{array}{l}\text { Ibuprofen } \\
800 \mathrm{mg}\end{array}$ & $\begin{array}{l}\text { Rapid response } \\
\text { to therapy with } \\
\text { quick symptom } \\
\text { improvement. }\end{array}$ & USA & No & Yes & Yes \\
\hline
\end{tabular}


Table 2. Cont.

\begin{tabular}{|c|c|c|c|c|c|c|c|c|c|c|c|c|c|c|c|c|}
\hline ID & Sex & Age & $\begin{array}{l}\text { Pre-Vaccine } \\
\text { History of } \\
\text { Immune } \\
\text { Mediated } \\
\text { Disease + } \\
\text { Medication }\end{array}$ & $\begin{array}{l}\text { Date 1st } \\
\text { Vaccine } \\
\text { Dose }\end{array}$ & $\begin{array}{l}\text { Date } \\
\text { 2nd } \\
\text { Vaccine } \\
\text { Dose }\end{array}$ & 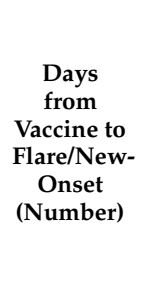 & $\begin{array}{c}\text { Immune } \\
\text { Mediated } \\
\text { Disease } \\
\text { Complication }\end{array}$ & $\begin{array}{l}\text { Flare } \\
\text { (F)/New- } \\
\text { Onset } \\
\text { (N) }\end{array}$ & Severity & $\begin{array}{l}\text { Relevant Lab } \\
\text { Tests }\end{array}$ & Therapy & $\begin{array}{l}\text { Comments on } \\
\text { Therapy and } \\
\text { Response }\end{array}$ & Country & $\begin{array}{c}\text { Can the } \\
\text { reaction } \\
\text { be } \\
\text { Explained } \\
\text { Based on } \\
\text { Drug } \\
\text { Received } \\
\text { by the } \\
\text { Patient? } \\
\text { (Yes/No) }\end{array}$ & $\begin{array}{c}\text { Can the } \\
\text { Reaction be } \\
\text { Explained } \\
\text { Based on the } \\
\text { Underlying } \\
\text { Co- } \\
\text { Morbidity } \\
\text { of the } \\
\text { Patient? } \\
\text { (Yes/No) }\end{array}$ & $\begin{array}{c}\text { Do you Feel } \\
\text { the Timing } \\
\text { of the } \\
\text { Side-Effect } \\
\text { Compatible } \\
\text { with the } \\
\text { Time from } \\
\text { Vaccine to } \\
\text { Flare? } \\
\text { (Yes/No) }\end{array}$ \\
\hline 10 & $\mathrm{~F}$ & 48 & Not relevant & $\begin{array}{l}\text { January } \\
2021 \\
\text { MRNA- } \\
1273\end{array}$ & $\begin{array}{l}\text { February } \\
2021\end{array}$ & $\begin{array}{l}10 \text { days } \\
\text { after 1st } \\
\text { dose }\end{array}$ & $\begin{array}{l}\text { Chilblains like } \\
\text { lesions on fingers } \\
\text { (painful) } \\
\text { Urticarial-type } \\
\text { lesions } \\
\text { (resembled } \\
\text { urticarial } \\
\text { multiforme). } \\
\text { 10 days after first } \\
\text { vaccine } \\
\text { developed itchy } \\
\text { urticarial lesions } \\
\text { over volar aspect } \\
\text { of both wrists } \\
\text { and feet, lesions } \\
\text { evolved over } \\
\text { thighs } \\
\text { (symptomless) } \\
\text { and extensor } \\
\text { surfaces of both } \\
\text { elbows, painful } \\
\text { macular/nodular } \\
\text { lesions over } \\
\text { palmar surface } \\
\text { both hands. } \\
\text { Resolved in } 7 \\
\text { days }\end{array}$ & $\mathrm{N}$ & Moderate & No work up & $\begin{array}{l}\text { Hydrocortisone } \\
0.5 \% \\
\\
\text { Naproxen } \\
500 \mathrm{mg}\end{array}$ & $\begin{array}{l}\text { Rapid response } \\
\text { to therapy with } \\
\text { quick symptom } \\
\text { improvement. }\end{array}$ & USA & No & No & Yes \\
\hline 11 & $\mathrm{M}$ & 21 & $\begin{array}{l}\text { Behcet's disease } \\
\text { (known for } 3 \\
\text { years) treated } \\
\text { with colchicine } \\
1.5 \mathrm{mg} \text { daily }\end{array}$ & $\begin{array}{c}\text { January } \\
2021 \\
\text { BNT162b2 } \\
\text { vaccine }\end{array}$ & $\begin{array}{c}\text { Not } \\
\text { given }\end{array}$ & $\begin{array}{l}5 \text { days } \\
\text { after 1st } \\
\text { dose }\end{array}$ & $\begin{array}{l}\text { Oral aphthous } \\
\text { ulcers }\end{array}$ & $\mathrm{F}$ & Moderate & No work up & $\begin{array}{l}20 \mathrm{mg} \text { of } \\
\text { prednisone } \\
\text { for } 1 \text { week }\end{array}$ & $\begin{array}{l}\text { Rapid response } \\
\text { to therapy with } \\
\text { quick symptom } \\
\text { improvement }\end{array}$ & Israel & No & No & Yes \\
\hline
\end{tabular}


Table 2. Cont.

\begin{tabular}{|c|c|c|c|c|c|c|c|c|c|c|c|c|c|c|c|c|}
\hline ID & Sex & Age & $\begin{array}{c}\text { Pre-Vaccine } \\
\text { History of } \\
\text { Immune } \\
\text { Mediated } \\
\text { Disease + } \\
\text { Medication }\end{array}$ & $\begin{array}{l}\text { Date 1st } \\
\text { Vaccine } \\
\text { Dose }\end{array}$ & $\begin{array}{l}\text { Date } \\
\text { 2nd } \\
\text { Vaccine } \\
\text { Dose }\end{array}$ & 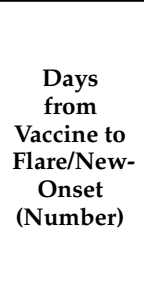 & $\begin{array}{c}\text { Immune } \\
\text { Mediated } \\
\text { Disease } \\
\text { Complication }\end{array}$ & $\begin{array}{l}\text { Flare } \\
\text { (F)/New- } \\
\text { Onset } \\
\text { (N) }\end{array}$ & Severity & $\begin{array}{l}\text { Relevant Lab } \\
\text { Tests }\end{array}$ & Therapy & $\begin{array}{l}\text { Comments on } \\
\text { Therapy and } \\
\text { Response }\end{array}$ & Country & $\begin{array}{c}\text { Can the } \\
\text { reaction } \\
\text { be } \\
\text { Explained } \\
\text { Based on } \\
\text { Drug } \\
\text { Received } \\
\text { by the } \\
\text { Patient? } \\
\text { (Yes/No) }\end{array}$ & $\begin{array}{c}\text { Can the } \\
\text { Reaction be } \\
\text { Explained } \\
\text { Based on the } \\
\text { Underlying } \\
\text { Co- } \\
\text { Morbidity } \\
\text { of the } \\
\text { Patient? } \\
\text { (Yes/No) }\end{array}$ & $\begin{array}{c}\text { Do you Feel } \\
\text { the Timing } \\
\text { of the } \\
\text { Side-Effect } \\
\text { Compatible } \\
\text { with the } \\
\text { Time from } \\
\text { Vaccine to } \\
\text { Flare? } \\
\text { (Yes/No) }\end{array}$ \\
\hline 12 & M & 55 & $\begin{array}{l}\text { Behcet's disease } \\
\text { known for } 20 \\
\text { years and well } \\
\text { controlled treated } \\
\text { with apremilast } \\
30 \mathrm{mg} \text { BID. Also } \\
\text { CLL (known for } 5 \\
\text { years, controlled } \\
\text { with no therapy) }\end{array}$ & $\begin{array}{l}\text { December } \\
2020 \\
\text { BNT162b2 } \\
\text { vaccine }\end{array}$ & $\begin{array}{l}\text { January } \\
2021 \\
\text { BNT162b2 } \\
\text { vaccine }\end{array}$ & $\begin{array}{l}7 \text { days } \\
\text { after } 2 \text { nd } \\
\text { dose }\end{array}$ & $\begin{array}{l}\text { Oral aphthous } \\
\text { ulcers and } \\
\text { synovitis of small } \\
\text { joints } \\
\text { (7 days after the } \\
\text { second dose, } \\
\text { synovitis in } \\
\text { MCPS and PIPS } \\
\text { both hands, and a } \\
\text { few days later } \\
\text { ulcers on the } \\
\text { tongue) }\end{array}$ & $\mathrm{F}$ & Moderate & No work up & $\begin{array}{c}\text { Colchicine } \\
0.5 \mathrm{mg} \text { twice } \\
\text { daily and } 5 \\
\mathrm{mg} \\
\text { prednisone }\end{array}$ & $\begin{array}{l}\text { Rapid response } \\
\text { to therapy with } \\
\text { quick symptom } \\
\text { improvement }\end{array}$ & Israel & No & No & Yes \\
\hline 13 & M & 20 & $\begin{array}{l}\text { Behcet's disease } \\
\text { (known for } 2 \\
\text { years) treated } \\
\text { with colchicine } 1 \\
\text { mg daily }\end{array}$ & $\begin{array}{l}\text { December } \\
2020 \\
\text { BNT162b2 } \\
\text { vaccine }\end{array}$ & $\begin{array}{c}\text { Not } \\
\text { given }\end{array}$ & $\begin{array}{l}3 \text { days } \\
\text { after 1st } \\
\text { dose }\end{array}$ & $\begin{array}{l}\text { Oral aphthous } \\
\text { ulcers }\end{array}$ & $\mathrm{F}$ & Moderate & No work up & $\begin{array}{l}\text { Increased } \\
\text { dosage of } \\
\text { colchicine to } \\
2 \mathrm{mg} \text { daily }\end{array}$ & $\begin{array}{l}\text { Rapid response } \\
\text { to therapy with } \\
\text { quick symptom } \\
\text { improvement }\end{array}$ & Israel & No & No & Yes \\
\hline 14 & F & 62 & $\begin{array}{c}\text { Patient with DM } \\
\text { treated with MTX } \\
\text { and PLQ } 200 \mathrm{mg} \\
\text { BID }\end{array}$ & $\begin{array}{l}\text { January } \\
2021 \\
\text { BNT162b2 } \\
\text { vaccine }\end{array}$ & $\begin{array}{l}\text { February } \\
2021 \\
\text { BNT162b2 } \\
\text { vaccine }\end{array}$ & $\begin{array}{l}7 \text { days } \\
\text { after 1st } \\
\text { dose }\end{array}$ & $\begin{array}{c}\text { Skin rash similar } \\
\text { to the rash when } \\
\text { diagnosed with } \\
\text { DM }\end{array}$ & $\mathrm{F}$ & Mild & No work up & $\begin{array}{l}\text { Local steroid } \\
\text { cream }\end{array}$ & $\begin{array}{c}\text { Flare } \\
\text { spontaneously } \\
\text { improved after a } \\
\text { week, the patient } \\
\text { received 2nd } \\
\text { dose of vaccine } \\
\text { with no } \\
\text { additional side } \\
\text { effects }\end{array}$ & Israel & No & No & Yes \\
\hline 15 & $\mathrm{~F}$ & 70 & $\begin{array}{l}\text { RA, treated with } \\
\text { MTX } 15 \text { mg wk } \\
\text { and } \\
\text { Certolizumab } 200 \\
\text { mg every other } \\
\text { week (on } \\
\text { remission for the } \\
\text { last } 18 \text { months). } \\
\text { Medical history } \\
\text { included } \\
\text { hypertension }\end{array}$ & $\begin{array}{l}\text { December } \\
2020 \\
\text { BNT162b2 } \\
\text { vaccine }\end{array}$ & $\begin{array}{l}\text { January } \\
2021 \\
\text { BNT162b2 } \\
\text { vaccine }\end{array}$ & $\begin{array}{l}7 \text { days } \\
\text { after } 2 \text { nd } \\
\text { dose }\end{array}$ & $\begin{array}{l}\text { Polyarthritis of } \\
\text { small joints }\end{array}$ & F & Moderate & High CRP & NSAIDs & $\begin{array}{l}\text { Rapid response } \\
\text { to therapy with } \\
\text { quick symptom } \\
\text { improvement }\end{array}$ & Israel & No & No & Yes \\
\hline
\end{tabular}


Table 2. Cont.

\begin{tabular}{|c|c|c|c|c|c|c|c|c|c|c|c|c|c|c|c|c|}
\hline ID & Sex & Age & $\begin{array}{l}\text { Pre-Vaccine } \\
\text { History of } \\
\text { Immune } \\
\text { Mediated } \\
\text { Disease + } \\
\text { Medication }\end{array}$ & $\begin{array}{l}\text { Date 1st } \\
\text { Vaccine } \\
\text { Dose }\end{array}$ & $\begin{array}{l}\text { Date } \\
\text { 2nd } \\
\text { Vaccine } \\
\text { Dose }\end{array}$ & $\begin{array}{l}\text { Days } \\
\text { from } \\
\text { Vaccine to } \\
\text { Flare/New- } \\
\text { Onset } \\
\text { (Number) }\end{array}$ & $\begin{array}{c}\text { Immune } \\
\text { Mediated } \\
\text { Disease } \\
\text { Complication }\end{array}$ & $\begin{array}{l}\text { Flare } \\
\text { (F)/New- } \\
\text { Onset } \\
\text { (N) }\end{array}$ & Severity & $\begin{array}{l}\text { Relevant Lab } \\
\text { Tests }\end{array}$ & Therapy & $\begin{array}{l}\text { Comments on } \\
\text { Therapy and } \\
\text { Response }\end{array}$ & Country & $\begin{array}{c}\text { Can the } \\
\text { reaction } \\
\text { be } \\
\text { Explained } \\
\text { Based on } \\
\text { Drug } \\
\text { Received } \\
\text { by the } \\
\text { Patient? } \\
\text { (Yes/No) }\end{array}$ & $\begin{array}{c}\text { Can the } \\
\text { Reaction be } \\
\text { Explained } \\
\text { Based on the } \\
\text { Underlying } \\
\text { Co- } \\
\text { Morbidity } \\
\text { of the } \\
\text { Patient? } \\
\text { (Yes/No) }\end{array}$ & $\begin{array}{c}\text { Do you Feel } \\
\text { the Timing } \\
\text { of the } \\
\text { Side-Effect } \\
\text { Compatible } \\
\text { with the } \\
\text { Time from } \\
\text { Vaccine to } \\
\text { Flare? } \\
\text { (Yes/No) }\end{array}$ \\
\hline 16 & F & 78 & $\begin{array}{l}\text { Laboratory SLE } \\
\text { with no clinical } \\
\text { manifestation }\end{array}$ & $\begin{array}{l}\text { January } \\
2021 \\
\text { BNT162b2 } \\
\text { vaccine }\end{array}$ & $\begin{array}{c}\text { Not } \\
\text { given }\end{array}$ & $\begin{array}{l}2 \text { days } \\
\text { after 1st } \\
\text { dose }\end{array}$ & $\begin{array}{l}\text { Fever, } \\
\text { erythematous } \\
\text { rash (generalized } \\
\text { acute cutaneous } \\
\text { lupus), purpura, } \\
\text { oral aphthous } \\
\text { ulcers and } \\
\text { arthritis }\end{array}$ & $\mathrm{N}$ & Mild & $\begin{array}{c}\text { +ANA, high } \\
\text { CRP and ESR, } \\
\text { Skin biopsy } \\
\text { from purpura } \\
\text { was } \\
\text { compatible } \\
\text { with leukocyto- } \\
\text { clastic } \\
\text { vasculitis }\end{array}$ & $\begin{array}{l}\text { Started on } \\
\text { Hydroxy- } \\
\text { chloroquine }\end{array}$ & $\begin{array}{l}\text { Rapid response } \\
\text { to therapy with } \\
\text { quick symptom } \\
\text { improvement }\end{array}$ & Israel & Yes & No & Yes \\
\hline 17 & $\mathrm{~F}$ & 50 & $\begin{array}{l}\text { SLE with } \\
\text { arthritis, mucosal } \\
\text { ulcers and } \\
\text { hemolysis (2019 } \\
\text { EULAR/ACR } \\
\text { SLE criteria) }\end{array}$ & $\begin{array}{l}\text { January } \\
2021 \\
\text { ChAdOx1 } \\
\text { nCoV-19 } \\
\text { vaccine }\end{array}$ & $\begin{array}{l}\text { Not } \\
\text { given }\end{array}$ & 14 & $\begin{array}{l}\text { Severe hemolysis, } \\
\text { oral and nasal } \\
\text { ulceration, } \\
\text { arthralgia }\end{array}$ & F & $\begin{array}{l}\text { Severe } \\
\text { (clinical as- } \\
\text { sessment) }\end{array}$ & $\begin{array}{c}\mathrm{Hb} 53, \\
\text { reticulocytes } \\
42 \% \text {, bilirubin } \\
48, \text { LDH } 864\end{array}$ & $\begin{array}{l}\text { High dose } \\
\text { glucocorti- } \\
\text { coids } \\
\text { (Pred- } \\
\text { nisolone } 60 \\
\text { mg daily) } \\
\text { and } \\
\text { rituximab }\end{array}$ & Slow response & UK & Yes & No & Yes \\
\hline 18 & M & 34 & $\begin{array}{l}\text { Behcet's disease } \\
\text { (known for } 10 \\
\text { years, included } \\
\text { arthritis, uveitis, } \\
\text { skin rash, oral } \\
\text { aphthosis } \\
\text { controlled for the } \\
\text { last } 12 \text { months, } \\
\text { treated with } \\
\text { colchicine and } \\
\text { Humira), no } \\
\text { other medical } \\
\text { history }\end{array}$ & $\begin{array}{c}\text { January } \\
2021 \\
\text { BNT162b2 } \\
\text { vaccine }\end{array}$ & $\begin{array}{l}\text { Not } \\
\text { given }\end{array}$ & $\begin{array}{l}5 \text { days } \\
\text { after } 1 \text { st } \\
\text { dose }\end{array}$ & $\begin{array}{l}\text { Pustular skin } \\
\text { lesions } \\
\text {-developed skin } \\
\text { lesions as a part } \\
\text { of Behcet's } \\
\text { disease for the } \\
\text { first time }\end{array}$ & F & Moderate & No work up & $\begin{array}{l}\text { Short course } \\
\text { of NSAIDs } \\
\text { and increase } \\
\text { of colchicine } \\
\text { dose }\end{array}$ & $\begin{array}{l}\text { Rapid response } \\
\text { to therapy with } \\
\text { quick symptom } \\
\text { improvement }\end{array}$ & Israel & No & No & Yes \\
\hline 19 & M & 72 & $\begin{array}{l}\text { Recurrent. } \\
\text { pericarditis - } \\
\text { treated with } \\
\text { colchicine in the } \\
\text { past }\end{array}$ & $\begin{array}{c}\text { January } \\
2021 \\
\text { BNT162b2 } \\
\text { vaccine }\end{array}$ & $\begin{array}{l}\text { January } \\
2021 \\
\text { BNT162b2 } \\
\text { vaccine }\end{array}$ & $\begin{array}{l}1 \text { day after } \\
\text { 2nd dose }\end{array}$ & $\begin{array}{l}\text { Myasthenia } \\
\text { Gravis }\end{array}$ & $\mathrm{N}$ & Moderate & $\begin{array}{l}\text { EMG- } \\
\text { decrement of } \\
28-46 \% \text { on } \\
\text { facial and } \\
\text { shoulder } \\
\text { muscles }\end{array}$ & $\begin{array}{l}\text { Plasma } \\
\text { exchange + } \\
\text { Prednisone } \\
\quad 60 \mathrm{mg}\end{array}$ & $\begin{array}{l}\text { Rapid response } \\
\text { to therapy with } \\
\text { quick symptom } \\
\text { improvement }\end{array}$ & Israel & No & No & Yes \\
\hline
\end{tabular}


Table 2. Cont

\begin{tabular}{|c|c|c|c|c|c|c|c|c|c|c|c|c|c|c|c|c|}
\hline ID & Sex & Age & $\begin{array}{l}\text { Pre-Vaccine } \\
\text { History of } \\
\text { Immune } \\
\text { Mediated } \\
\text { Disease + } \\
\text { Medication }\end{array}$ & $\begin{array}{l}\text { Date 1st } \\
\text { Vaccine } \\
\text { Dose }\end{array}$ & $\begin{array}{l}\text { Date } \\
\text { 2nd } \\
\text { Vaccine } \\
\text { Dose }\end{array}$ & $\begin{array}{l}\text { Days } \\
\text { from } \\
\text { Vaccine to } \\
\text { Flare/New- } \\
\text { Onset } \\
\text { (Number) }\end{array}$ & $\begin{array}{c}\text { Immune } \\
\text { Mediated } \\
\text { Disease } \\
\text { Complication }\end{array}$ & $\begin{array}{l}\text { Flare } \\
\text { (F)/New- } \\
\text { Onset } \\
\text { (N) }\end{array}$ & Severity & $\begin{array}{c}\text { Relevant Lab } \\
\text { Tests }\end{array}$ & Therapy & $\begin{array}{l}\text { Comments on } \\
\text { Therapy and } \\
\text { Response }\end{array}$ & Country & $\begin{array}{l}\text { Can the } \\
\text { reaction } \\
\text { be } \\
\text { Explained } \\
\text { Based on } \\
\text { Drug } \\
\text { Received } \\
\text { by the } \\
\text { Patient? } \\
\text { (Yes/No) }\end{array}$ & $\begin{array}{c}\text { Can the } \\
\text { Reaction be } \\
\text { Explained } \\
\text { Based on the } \\
\text { Underlying } \\
\text { Co- } \\
\text { Morbidity } \\
\text { of the } \\
\text { Patient? } \\
\text { (Yes/No) }\end{array}$ & $\begin{array}{c}\text { Do you Feel } \\
\text { the Timing } \\
\text { of the } \\
\text { Side-Effect } \\
\text { Compatible } \\
\text { with the } \\
\text { Time from } \\
\text { Vaccine to } \\
\text { Flare? } \\
\text { (Yes/No) }\end{array}$ \\
\hline 20 & M & 73 & Not relevant & $\begin{array}{l}\text { Dec } 2020 \\
\text { BNT162b2 } \\
\text { vaccine }\end{array}$ & $\begin{array}{l}\text { January } \\
2021 \\
\text { BNT162b2 } \\
\text { vaccine }\end{array}$ & $\begin{array}{l}7 \text { days } \\
\text { after } 2 \text { nd } \\
\text { dose }\end{array}$ & $\begin{array}{l}\text { Myasthenia } \\
\text { Gravis (firstly } \\
\text { ocular signs then } \\
\text { appearance of } \\
\text { bulbar signs and } \\
\text { respiratory } \\
\text { symptoms) }\end{array}$ & $\mathrm{N}$ & Severe & $\begin{array}{l}\text { EMG- } \\
\text { borderline } \\
\text { decrement, } \\
\text { markedly } \\
\text { pathologic } \\
\text { jitter }\end{array}$ & $\begin{array}{l}\text { Plasma } \\
\text { Exchange, } \\
\text { Pyridostig- } \\
\text { min and } \\
\text { prednisone }\end{array}$ & $\begin{array}{l}\text { Patient intubated } \\
\text { due to respiratory } \\
\text { symptoms }\end{array}$ & Israel & No & No & Yes \\
\hline 21 & $\mathrm{~F}$ & 68 & $\begin{array}{l}\text { RA } \\
\text { seropositive-well } \\
\text { controlled with } \\
\text { Actemra }\end{array}$ & $\begin{array}{l}\text { January } \\
2021 \\
\text { BNT162b2 } \\
\text { vaccine }\end{array}$ & $\begin{array}{l}\text { February } \\
2021 \\
\text { BNT162b2 } \\
\text { vaccine }\end{array}$ & $\begin{array}{l}3 \text { days } \\
\text { after } 2 \text { nd } \\
\text { dose }\end{array}$ & $\begin{array}{l}\text { Flare of synovitis } \\
\text { of small joints } \\
\text { CDAI }=41 \\
\text { compared to } \\
\text { CDAI }=4 \text { in } 19 \\
\text { January } 2021\end{array}$ & $\mathrm{~F}$ & Severe & $\begin{array}{c}\text { ESR=12 after } \\
\text { depomedrol, } \\
C R P=0.06 \\
\text { before flare, } \\
C R P=0.28 \\
\text { after flare, } R F= \\
1782 \text { before } \\
\text { flare, } R F=2300 \\
\text { after flare, } \\
\text { ANA negative } \\
\text { after vaccine }\end{array}$ & $\begin{array}{c}\text { IM } \\
\text { Depomedrol } \\
160 \mathrm{mg}\end{array}$ & $\begin{array}{l}\text { Rapid response } \\
\text { to therapy with } \\
\text { quick symptom } \\
\text { improvement }\end{array}$ & Israel & No & No & Yes \\
\hline 22 & M & 53 & Not relevant & $\begin{array}{l}\text { January } \\
2021 \\
\text { BNT162b2 } \\
\text { vaccine }\end{array}$ & $\begin{array}{l}\text { Not } \\
\text { given }\end{array}$ & $\begin{array}{l}3 \text { days } \\
\text { after first } \\
\text { dose }\end{array}$ & $\begin{array}{c}\text { Palpable purpura, } \\
\text { mild abdominal } \\
\text { pain and } \\
\text { arthralgia w/o } \\
\text { arthritis, no renal } \\
\text { involvement. } \\
\text { Diagnosed with } \\
\text { Henoch } \\
\text { Schlonein Pupura } \\
\text { with } \\
\text { Leukocytoclastic } \\
\text { cutaneous } \\
\text { vasculitis among } \\
\text { skin bipsy }\end{array}$ & $\mathrm{N}$ & $\begin{array}{c}\text { Mild- } \\
\text { moderate }\end{array}$ & $\begin{array}{l}\text { Skin biopsy } \\
\text { revealed IgA } \\
\text { and C3 } \\
\text { deposits in the } \\
\text { vessel walls } \\
\text { Mild elevation } \\
\text { in CRP to } 0.8 \\
\mathrm{mg} / \mathrm{dL} \text {, mild } \\
\text { elevation in } \\
\mathrm{IgA} \text { to } 412 \\
\mathrm{mg} \% \text {, mild } \\
\text { leucocytosis to } \\
11.5 \mathrm{k} / \mathrm{ul}\end{array}$ & $\begin{array}{l}\text { Dexamethasone } \\
10 \mathrm{mg}, \\
\text { prednisone } \\
60 \mathrm{mg} \\
\text { thereafter }\end{array}$ & $\begin{array}{l}\text { Rapid response } \\
\text { to therapy with } \\
\text { quick symptom } \\
\text { improvement }\end{array}$ & Israel & No & No & Yes \\
\hline
\end{tabular}


Table 2. Cont.

\begin{tabular}{|c|c|c|c|c|c|c|c|c|c|c|c|c|c|c|c|c|}
\hline ID & Sex & Age & $\begin{array}{l}\text { Pre-Vaccine } \\
\text { History of } \\
\text { Immune } \\
\text { Mediated } \\
\text { Disease + } \\
\text { Medication }\end{array}$ & $\begin{array}{l}\text { Date 1st } \\
\text { Vaccine } \\
\text { Dose }\end{array}$ & $\begin{array}{l}\text { Date } \\
\text { 2nd } \\
\text { Vaccine } \\
\text { Dose }\end{array}$ & $\begin{array}{l}\text { Days } \\
\text { from } \\
\text { Vaccine to } \\
\text { Flare/New- } \\
\text { Onset } \\
\text { (Number) }\end{array}$ & $\begin{array}{c}\text { Immune } \\
\text { Mediated } \\
\text { Disease } \\
\text { Complication }\end{array}$ & $\begin{array}{l}\text { Flare } \\
\text { (F)/New- } \\
\text { Onset } \\
\text { (N) }\end{array}$ & Severity & $\begin{array}{l}\text { Relevant Lab } \\
\text { Tests }\end{array}$ & Therapy & $\begin{array}{l}\text { Comments on } \\
\text { Therapy and } \\
\text { Response }\end{array}$ & Country & $\begin{array}{c}\text { Can the } \\
\text { reaction } \\
\text { be } \\
\text { Explained } \\
\text { Based on } \\
\text { Drug } \\
\text { Received } \\
\text { by the } \\
\text { Patient? } \\
\text { (Yes/No) }\end{array}$ & $\begin{array}{c}\text { Can the } \\
\text { Reaction be } \\
\text { Explained } \\
\text { Based on the } \\
\text { Underlying } \\
\text { Co- } \\
\text { Morbidity } \\
\text { of the } \\
\text { Patient? } \\
\text { (Yes/No) }\end{array}$ & $\begin{array}{c}\text { Do you Feel } \\
\text { the Timing } \\
\text { of the } \\
\text { Side-Effect } \\
\text { Compatible } \\
\text { with the } \\
\text { Time from } \\
\text { Vaccine to } \\
\text { Flare? } \\
\text { (Yes/No) }\end{array}$ \\
\hline 23 & $\mathrm{~F}$ & 80 & $\begin{array}{c}\text { Seronegative } \\
\text { RA-2007 } \\
\text { (ACR/EULAR } \\
\text { 2010 class. Crit.) } \\
\text { stable on } \\
\text { Methotrexate } \\
\text { 15mgs week }\end{array}$ & $\begin{array}{l}\text { January } \\
2021 \\
\text { BNT162b2 } \\
\text { vaccine }\end{array}$ & $\begin{array}{l}\text { Not } \\
\text { given }\end{array}$ & $\begin{array}{l}25 \text { days } \\
\text { flare with } \\
\text { progres- } \\
\text { sive } \\
\text { worsening }\end{array}$ & $\begin{array}{l}\text { Severe Right } \\
\text { ankle synovitis, } \\
\text { severe wrist } \\
\text { synovitis and } 5 \\
\text { small joint } \\
\text { synovitis }\end{array}$ & $\mathrm{F}$ & $\begin{array}{l}\text { Severe } \\
\text { (articular } \\
\text { swelling } \\
\text { and sub- } \\
\text { stantial } \\
\text { limitation } \\
\text { of range of } \\
\text { motion) }\end{array}$ & $\begin{array}{l}\text { ESR } 71 \\
\text { CRP } 27\end{array}$ & $\begin{array}{l}\text { Prednisolone } \\
15 \text { mgs day } \\
\text { tapering and } \\
\text { increase } \\
\text { methotrex- } \\
\text { ate } \\
\text { dose }\end{array}$ & $\begin{array}{l}\text { Rapid response } \\
\text { to therapy with } \\
\text { quick symptom } \\
\text { improvement }\end{array}$ & UK & No & No & Yes \\
\hline 24 & $\mathrm{M}$ & 70 & $\begin{array}{l}\text { Gout (known for } \\
\text { many years) well } \\
\text { controlled in the } \\
\text { last } 2 \text { years), } \\
\text { hypertension and } \\
\text { dyslipidaemia }\end{array}$ & $\begin{array}{c}\text { Dec } 2020 \\
\text { BNT162b2 } \\
\text { vaccine }\end{array}$ & $\begin{array}{l}\text { January } \\
2021 \\
\text { BNT162b2 } \\
\text { vaccine }\end{array}$ & $\begin{array}{l}1 \text { day after } \\
\text { first dose } \\
\text { and } \\
\text { another } \\
\text { flare } 1 \text { day } \\
\text { after the } \\
\text { second } \\
\text { dose }\end{array}$ & $\begin{array}{l}\text { Monoarthritis } \\
\text { at Rt elbow (after } \\
\text { 1st dose) and at } \\
\text { mid foot (after } \\
\text { 2nd dose) }\end{array}$ & F & Moderate & CRP 44 mg/L. & $\begin{array}{l}\text { Prednisone } \\
40 \mathrm{mg} \text { for } \\
7 \text { day }\end{array}$ & $\begin{array}{l}\text { Rapid response } \\
\text { to therapy with } \\
\text { quick symptom } \\
\text { improvement }\end{array}$ & Israel & No & No & Yes \\
\hline 25 & $\mathrm{~F}$ & 78 & $\begin{array}{l}\text { Seropositive RA } \\
\text { (not active and } \\
\text { with no therapy } \\
\text { for many years) }\end{array}$ & $\begin{array}{l}\text { January } \\
2021 \\
\text { BNT162b2 } \\
\text { vaccine }\end{array}$ & $\begin{array}{l}\text { Not } \\
\text { given }\end{array}$ & $\begin{array}{l}2 \text { days } \\
\text { after first } \\
\text { dose }\end{array}$ & $\begin{array}{l}\text { Polyarthritis } \\
\text { involving } \\
\text { shoulders, wrists, } \\
\text { MCPs and PIPs }\end{array}$ & F & Moderate & $\begin{array}{l}\text { ANA negative, } \\
\text { RF 60, ESR 52, } \\
\text { CRP } 2.1 \mathrm{mg} / \mathrm{dl}\end{array}$ & $\begin{array}{l}\text { Prednisone } \\
20 \mathrm{mg} \text { daily } \\
\text { with } \\
\text { tapering } \\
\text { down } 5 \mathrm{mg} \\
\text { every week } \\
\text { was } \\
\text { prescribed. }\end{array}$ & $\begin{array}{l}\text { Rapid response } \\
\text { to therapy with } \\
\text { quick symptom } \\
\text { improvement }\end{array}$ & Israel & No & No & Yes \\
\hline 26 & $\mathrm{~F}$ & 27 & $\begin{array}{c}\text { Seronegative RA } \\
\text { stable on } \\
\text { Adalimumab } \\
\text { every } 2 \text { weeks }\end{array}$ & $\begin{array}{l}\text { January } \\
2021 \\
\text { BNT162b2 } \\
\text { vaccine }\end{array}$ & $\begin{array}{l}\text { January } \\
2021 \\
\text { BNT162b2 } \\
\text { vaccine }\end{array}$ & $\begin{array}{l}4 \text { days } \\
\text { after } \\
\text { second } \\
\text { dose } \\
\text { developed } \\
\text { Rt knee } \\
\text { monoarthri- } \\
\text { tis }\end{array}$ & $\begin{array}{l}\text { Monoarthritis of } \\
\text { Rt knee }\end{array}$ & $\mathrm{F}$ & Moderate & $\begin{array}{l}\text { ANA negative, } \\
\text { RF negative, } \\
\text { CRP } 1.3 \mathrm{mg} / \mathrm{dl}\end{array}$ & $\begin{array}{c}\text { Joint } \\
\text { aspiration } \\
\text { and injection } \\
\text { of } 80 \mathrm{mg} \\
\text { depomedrol }\end{array}$ & $\begin{array}{l}\text { Rapid response } \\
\text { to therapy with } \\
\text { quick symptom } \\
\text { improvement }\end{array}$ & Israel & No & No & Yes \\
\hline
\end{tabular}


Table 2. Cont

\begin{tabular}{|c|c|c|c|c|c|c|c|c|c|c|c|c|c|c|c|c|}
\hline ID & Sex & Age & $\begin{array}{l}\text { Pre-Vaccine } \\
\text { History of } \\
\text { Immune } \\
\text { Mediated } \\
\text { Disease + } \\
\text { Medication }\end{array}$ & $\begin{array}{l}\text { Date 1st } \\
\text { Vaccine } \\
\text { Dose }\end{array}$ & $\begin{array}{c}\text { Date } \\
\text { 2nd } \\
\text { Vaccine } \\
\text { Dose }\end{array}$ & 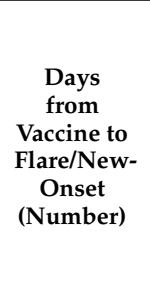 & $\begin{array}{c}\text { Immune } \\
\text { Mediated } \\
\text { Disease } \\
\text { Complication }\end{array}$ & $\begin{array}{l}\text { Flare } \\
\text { (F)/New- } \\
\text { Onset } \\
\text { (N) }\end{array}$ & Severity & $\begin{array}{l}\text { Relevant Lab } \\
\text { Tests }\end{array}$ & Therapy & $\begin{array}{l}\text { Comments on } \\
\text { Therapy and } \\
\text { Response }\end{array}$ & Country & $\begin{array}{l}\text { Can the } \\
\text { reaction } \\
\text { be } \\
\text { Explained } \\
\text { Based on } \\
\text { Drug } \\
\text { Received } \\
\text { by the } \\
\text { Patient? } \\
\text { (Yes/No) }\end{array}$ & $\begin{array}{c}\text { Can the } \\
\text { Reaction be } \\
\text { Explained } \\
\text { Based on the } \\
\text { Underlying } \\
\text { Co- } \\
\text { Morbidity } \\
\text { of the } \\
\text { Patient? } \\
\text { (Yes/No) }\end{array}$ & $\begin{array}{c}\text { Do you Feel } \\
\text { the Timing } \\
\text { of the } \\
\text { Side-Effect } \\
\text { Compatible } \\
\text { with the } \\
\text { Time from } \\
\text { Vaccine to } \\
\text { Flare? } \\
\text { (Yes/No) }\end{array}$ \\
\hline 27 & F & 60 & $\begin{array}{l}\text { SLE (never } \\
\text { previously } \\
\text { referred to } \\
\text { rheumatology): } \\
\text { Discoid lupus } \\
\text { since 2001, severe } \\
\text { raynauds, } \\
\text { alopecia, } \\
\text { pancytopenia } \\
\text { (since September } \\
\text { 2020), proteinuria } \\
\text { (Developed Sept } \\
\text { 2020, diagnosed } \\
\text { as class IV lupus } \\
\text { nephritis March } \\
\text { 2021), hypocom- } \\
\text { plementaemia, } \\
\text { ANA positive, } \\
\text { dsDNA, } \\
\text { anti-chromatin } \\
\text { and } \\
\text { anti-ribosomal } \\
\text { antibody } \\
\text { positive), 2019 } \\
\text { EULAR/ACR } \\
\text { SLE criteria) } \\
\text { Baseline Disease } \\
\text { modifying } \\
\text { therapy: } \\
\text { Prednisolone } \\
\text { 8mg OD }\end{array}$ & $\begin{array}{l}\text { February } \\
2021 \\
\text { ChAdOx1 } \\
\text { vaccine }\end{array}$ & $\begin{array}{l}\text { Not } \\
\text { given } \\
\text { yet }\end{array}$ & 4 days & $\begin{array}{l}\text { Pericardial and } \\
\text { pleural effusions, } \\
\text { possible } \\
\text { pericarditis }\end{array}$ & F & Mild & $\begin{array}{c}\text { Central } \\
\text { intermittent } \\
\text { pleuritic chest } \\
\text { pain, had } \\
\text { CTPA and } \\
\text { bedside echo. } \\
\text { Trop normal } \\
\text { CRP 17.8 }\end{array}$ & $\begin{array}{c}\text { Prednisone } \\
\text { increased } \\
\text { from } 8 \mathrm{mg} \text { to } \\
15 \mathrm{mg} \text { for } 5 \\
\text { days, } \\
\text { colchicine } \\
500 \text { mcg BD }\end{array}$ & $\begin{array}{l}\text { Rapid response } \\
\text { to therapy with } \\
\text { quick symptom } \\
\text { improvement }\end{array}$ & UK & Yes & Yes & Yes \\
\hline
\end{tabular}




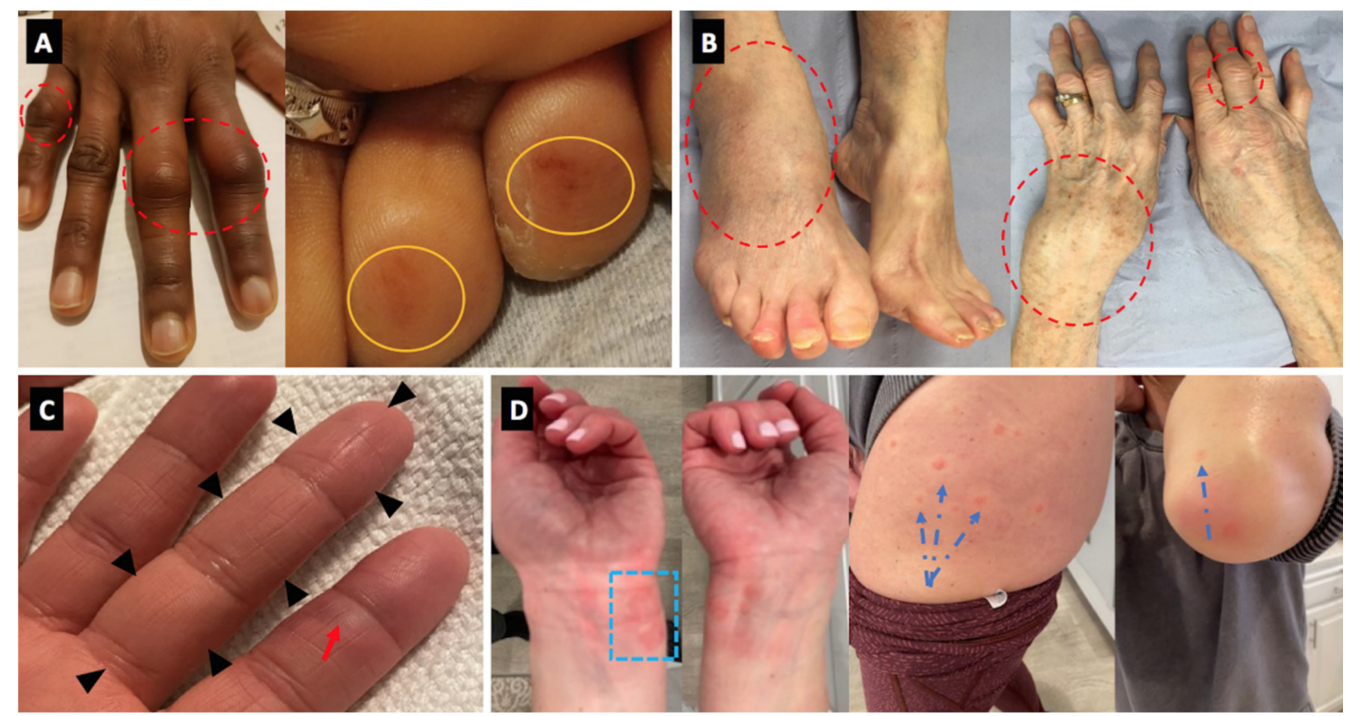

Figure 2. Panel (A) (Case 2)-Florid clinical arthritis of the proximal interphalangeal joints (dotted red circles). Concomitant, painless rash on two toe tips (orange circles). Panel (B) (Case 23)—Severe articular swelling of the right ankle, left wrist, and the small joints of the fingers (dotted red circles). Panel (C) (Case 9)-dactylitis of the third finger (black arrowheads) associated with chilblain lesion of the second finger (red arrow). Panel (D) (Case 10)—chilblain-like lesions of the fingers and palms, associated with urticarial lesions of the wrists (blue dotted square), thighs and elbows (blue dotted arrows).
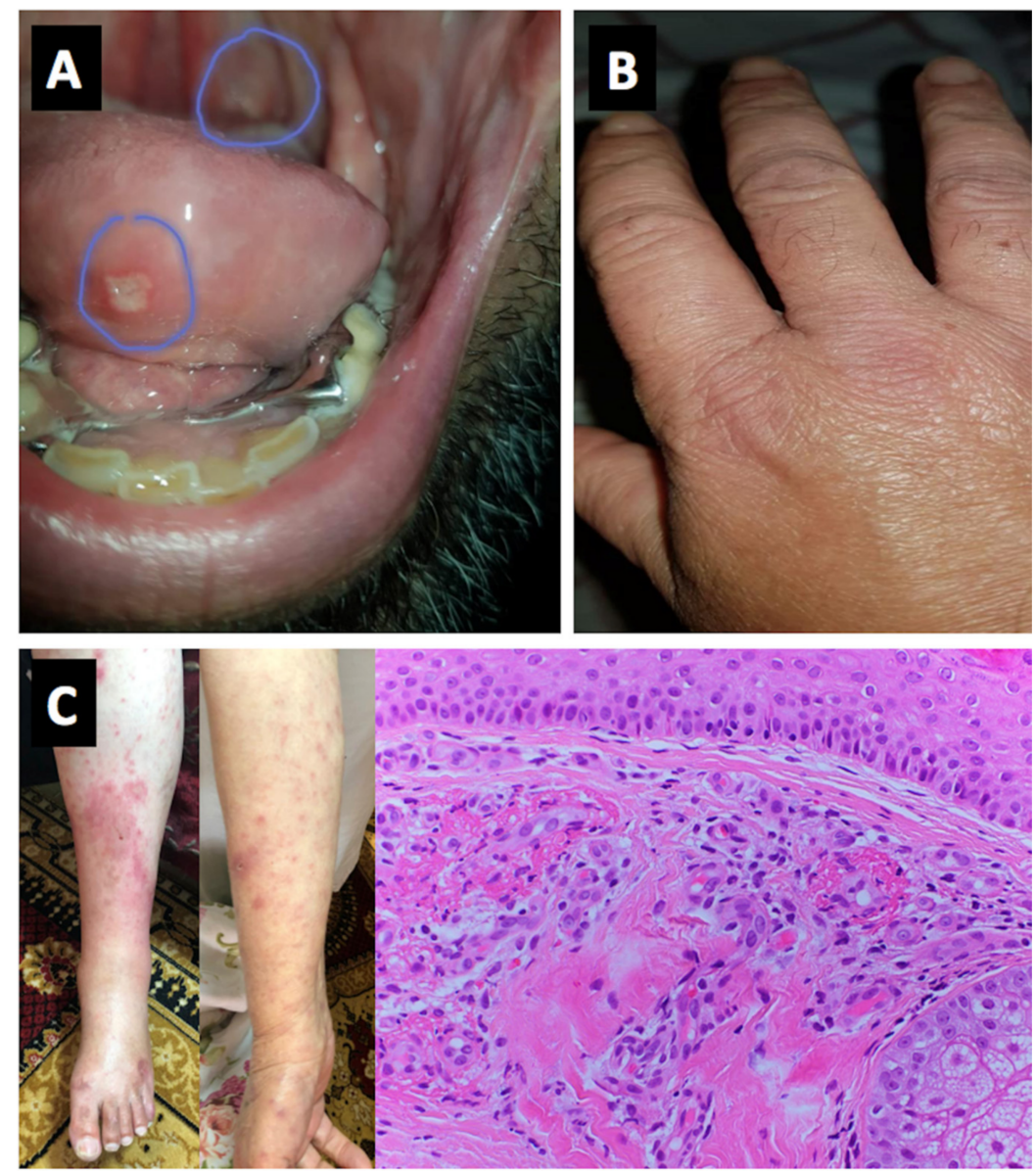

Figure 3. Cont. 


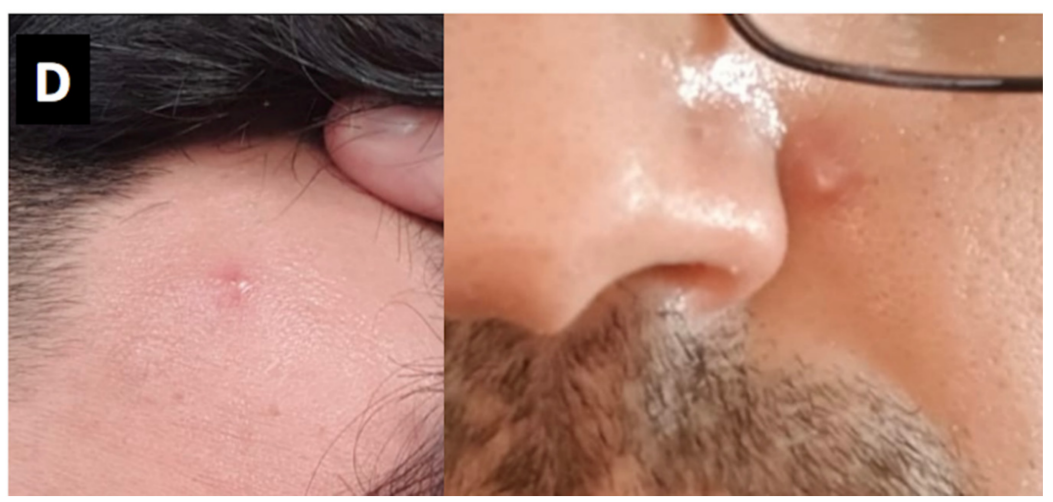

Figure 3. Panels (A,B) (Cases 11 and 12)—Oral aphthous lesions and small joints arthritis, Behçet's disease. Panel (C) (Case 22) - Vasculitic lesions of the skin on lower limbs and forearms. Histology consistent with fibrinoid necrosis of small vessels. Panel (D) (Case 17)—Pustular skin lesions on the forehead and face, Behçet's disease.

\subsection{Laboratory Findings}

Among 11 cases of arthritis, only 3 were of autoimmune (RF was positive in two cases and 1 was anti-nuclear antibody or ANA positive). ACPA was positive in only one case of pericarditis but with no particular manifestation. CRP and ESR were generally high. Other laboratory tests are reported in Table 1.
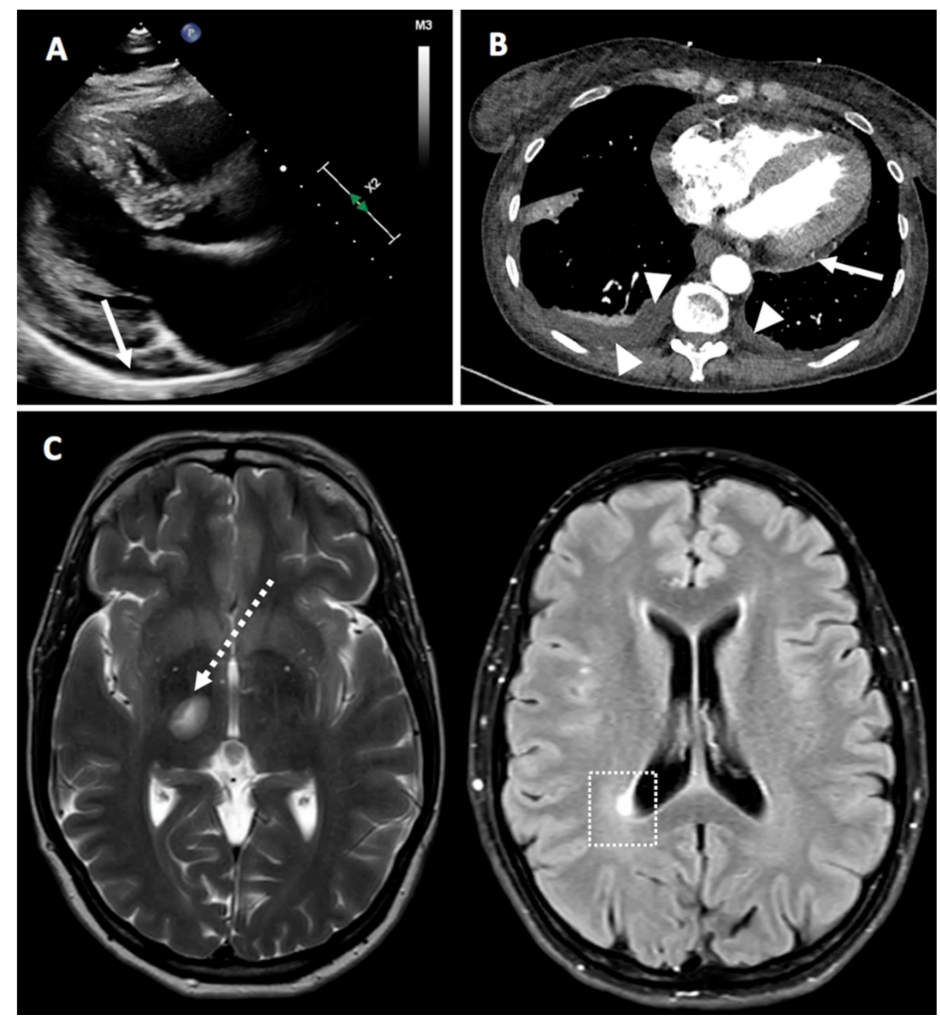

Figure 4. Panel (A) (Case 4) -Parasternal, long axis view from a 2-D echocardiogram showing a smallsized posterior pericardial effusion (white arrow). Panel (B) (Case 27)—Computerized tomography scan demonstrating posterior pericardial effusion (white arrow) and bilateral pleural effusion (white arrowheads). Panel (C) (Case 7)—Magnetic resonance imaging of the brain showing demyelinating lesions in the left mesencephalic (dotted white arrow) and in the right peri-ventricular occipital white matter (white dotted square). 


\subsection{Therapy}

As outlined in Tables 1 and 2, the majority of cases 21 (80.8\%) received glucocorticoid therapy. Other drugs included colchicine, NSAIDs, hydroxychloroquine, rituximab, plasma exchange and pyridostigmine. At the time of writing no patient has severe resistant or progressive disease with all cases showing good therapy responses.

\section{Discussion}

Natural viral infection or vaccination have both been noted as potential triggering events for inflammatory diseases for many decades [40,41]. Herein, we combined data from three countries from large academic centers in regions where anti SARS-CoV-2 vaccination programs rolled out with an estimate catchment population of 6-7 million subjects. We report the nature of flares in known IMDs that generally responded well to corticosteroid therapy. We reported less common, but notable, new-onset IMDs temporally associated with vaccination administration, including neurological disease, which represents the main strength of the present investigation.

Overall, these findings of new-onset disease appear rare considering the level of population exposure in the regions covered and are likely to be close to the background incidence of these conditions in such a large population. While we cannot estimate frequency with precision, the level of public and medical vigilance around this new healthcare intervention would suggest that cases were less likely to be missed compared to, for example, seasonal influenza. Further studies would be required to compare the frequencies of IMDs after these vaccines.

Despite being rare, the insurgence of AEFIs represents a public health concern in that, if not dealt with rapidly and properly, they could undermine public confidence and trust towards vaccination. COVID-19 immunization seems to be extremely a valid strategy to contain and mitigate against the burden imposed by the pandemic and, in order to reach herd immunity, high compliance rates are warranted. Acceptance rate towards COVID-19 immunization is highly variable, ranging from approximately $90 \%$ to less than $55 \%$, depending on the country, with information being disseminated and received playing a major role in achieving optimal coverage and community immunity [42]. Therefore, COVID-19 vaccine safety assessment is extremely crucial, especially at the beginning of population-level implementation. Our data assist clinicians in recognizing putative flares that are in line with our reported experience. The outcomes provide assurance to the public that such events are rare and manageable, and to clinicians that conventional therapies such as glucocorticoids are usually adequate-particularly important at a time where healthcare resources are highly stretched.

Numerically, our findings show the clearest associations for the non-specific adjuvant effects of the mRNA vaccines in triggering a host of different inflammatory disorders. It is possible that there is increased vigilance towards, and documentation of non-arthritis RMDs, since these are more likely to require specialist review, other investigations, or hospital treatment. Nevertheless, the proportion of the events observed is disproportionate to the usual frequency of these diseases. Usual prevalence would predict RA most commonly, followed by other inflammatory arthritis, and, then, non-arthritis RMDS. Here, we found that almost half of the events occur as forms of non-arthritis RMDs-usually far less frequent than inflammatory arthritis. Within inflammatory arthritis, spondyloarthropathyspectrum disease was more frequent than RA, again differing from the usual prevalence, while one RA case had a usually-rare extra-articular feature. This suggests, although does not prove, causality. Most of the reported disease were flares, which supports the idea of the delicate balance of immune homeostasis in such cases being momentarily tipped into a pro-inflammatory state by vaccination.

The majority of patients in our study received mRNA vaccines while only two received DNA-based vaccine. This may simply reflect the roll-out of these vaccines rather than a causal association with IMDs. However, of particular importance, the use of TLR-7 and TLR-9 agonistic based vaccines which stimulates immunity in different ways to the 
more TLR-4 and inflammasome based alum vaccines represents a new vaccine strategy for human disease [43]. Most of our cases, but not all, responded very well to therapy and in most cases second doses of vaccines were withheld.

Natural SARS-CoV-2 infection has resulted so far in over 3.1 million deaths due to severe viral pneumonia where immune hyper-activation also contributes to mortality as shown by the response to some patients to corticosteroids. The most well-recognized RMD-like feature of natural SARS-CoV-2 infection is the various cutaneous vasculitislike feature including "COVID toes" and the Multisystem Inflammatory Syndrome in Children (MIS-C) [44]. Of note, these manifestations are generally seen in subjects with minimal or not lung disease pointing towards a hyperactive immune response which, while being protective against pneumonia, can lead to collateral damage to other organ systems. Natural infection has also been associated with case reports of vasculitis, neurological disease including GBS and others in addition to other occasionally reported IMDs [45-47]. Herein, we report a wide variety of vaccine-associated flares or new-onset in IMDs ranging from autoinflammatory, mixed pattern disease (BD) and autoimmune diseases rather than an enrichment in autoimmune diseases that are linked to disordered nucleic acid metabolism and abnormal interferon-stimulated gene signatures involving the TLR-7/9 pathways [43].

Our findings show the presence of concomitant inflammatory arthritis and usual skin rashes in some cases. It is well established that younger subjects in particular, without severe COVID-19, experienced chilblain and erythematous lesions of the toes which had associated elevations in Type-I interferon in the skin contributing to a type of vasculitis. Of note, the TLR-7 and TLR-9 stimulation afforded by these new generations of vaccines might be expected to upregulate interferon-stimulated genes (ISGs) and contribute to robust early innate immune responses with robust type-I IFN responses thus accounting for the cutaneous and potentially other features. Dysregulated nucleic acid metabolism is associated with interferonopathies and SLE and other ANA-associated phenotypes. The frequency of such diseases is low in our series, although being similar to our rate of ACPA-positive RA this may be considered disproportionately high. We noted one case of discoid SLE converting into a systemic phenotype, with pleuro-pericarditis shortly after the TLR-9 agonist containing DNA vaccine, one case of SLE that had low activity, but where rituximab was deferred, to enhance vaccine efficacy and where patient flared following the DNA vaccine and a third case of dermatomyositis rash flare following an RNA vaccine. Monogenic interferonopathies are known to often include chilblains. Reassuringly, autoimmune myositis was not documented which is noteworthy given the intramuscular route of vaccine administration. Other relevant negatives were an absence of Macrophage Activation Syndrome (MAS)-like patterns of disease such as AdultOnset Still's Disease (AOSD) and undefined hyperinflammatory states mimicking MIS-C, although it is acknowledged that the latter is a disease of children and young adults, none of whom were vaccinated in the present study.

In the existing literature, only few anecdotal side-effects have been reported, including oral, oro-facial, and allergic reactions [48-50]. Of note, cases of Bell's palsy, and swelling of the lips, face or tongue associated with anaphylaxis as well as flares of RA were reported [50,51]

In addition to the non-specific vaccine adjuvant properties triggered reactions as discussed above [52-55], anaphylactic reactions to BNT162b2 and mRNA-1273 based vaccines have been reported, allegedly attributed to adjuvants, like the polyethylene glycol (PEG) 2000 present in the lipid film of the two vaccine products or the polysorbate 80 utilized as excipient formulation in the preparation of the ChAdOx1 nCoV-19 vaccine [48-50].

In the present study, we also noted flares in BD. However, elevations in IFN levels and especially the use of recombinant IFN-alpha may have a therapeutic role in $\mathrm{BD}$, although the mechanism of action of IFN-alpha in BD is not fully understood and there may be pathogenic heterogeneity.

Despite its strength, our study has several limitations, that should be properly acknowledged. From the design of this non-systematic accrual and reporting of IMDs in this 
early phase of the COVID-19 vaccine roll-out it is impossible to compute AEFI incidence rate or be certain of causality, even though the number of cases, their unusual presentations and apparent de-challenge response from the time of vaccine administration raise the possibility of their relationship. Other possibilities include that some of the cases actually could have had concomitant SARS-CoV-2 infection but the clinical picture without respiratory symptoms does not support this. It is also possible that some cases had prior COVID-19 disease which could have contributed to immune responses and we did not test for prior SARS-CoV-2 immunity as assessed by antibody responses. Summarizing, multiple confounding factors including concomitant SARS-CoV-2 infection and multiple co-morbidities in some cases may be contributory factors to the observed symptomatology. Given the nature of the data collection it is impossible to know how common IMD new-onset or flare of existing IMDs are and it is possible that these findings are coincidental. Nevertheless, some unusual features including concomitant rashes with IMDs that have been reported with SARS-CoV-2 infection suggest a link between vaccination, at least, in some subjects.

In conclusion this is the first large series description of IMDs flares or new-onset temporally associated with SARS-CoV-2 vaccination. The majority of cases had disease that quickly settled with corticosteroid therapy. Larger high-quality, prospective systematic studies are needed to address the issue of COVID-19 vaccination. Finally, we did not come across any cases of suspected immunothrombosis although we interacted with haematologists but it is noteworthy that $25 / 27$ cases received mRNA vaccines.

Author Contributions: Conceptualization, A.W. and D.M.; methodology, N.L.B.; formal analysis, N.L.B.; investigation, A.W. and D.M.; Data Collection, A.W. and D.M.; Data Collection, A.W., G.D.M., H.M., A.D., M.E. (Mailam Eltity), N.H., A.H., M.E. (Muna Elisa), D.Z., Y.C., A.A.-M., M.A.E., C.B., P.L., J.M., N.L.B., H.M.-O., M.L., C.C., L.C., E.V., Y.S., H.A., and D.M.; data curation, A.W. and D.M.; Data Collection, A.W., G.D.M., H.M., A.D., M.E. (Mailam Eltity), N.H., A.H., M.E. (Muna Elisa), M.E.N., M.B., D.Z., Y.C., A.A.-M., M.A.E., C.B., P.L., J.M., N.L.B., H.M.-O., M.L., C.C., L.C., E.V., Y.S., H.A., and D.M.; writing-original draft preparation, A.W., N.L.B., H.A., and D.M.; writing, editing and reviewing-A.W. and D.M.; Data Collection, A.W., G.D.M., H.M., A.D., M.E. (Mailam Eltity), N.H., A.H., M.E. (Muna Elisa), M.E.N., M.B., D.Z., Y.C., A.A.-M., M.A.E., C.B., P.L., J.M., N.L.B., H.M.-O., M.L., C.C., L.C., E.V., Y.S., H.A., and D.M.; supervision, A.W., H.A., and D.M.; funding acquisition, D.M. All authors have read and agreed to the published version of the manuscript.

Funding: This research received no external funding.

Informed Consent Statement: Informed, written consent was obtained from all subjects involved in the study.

Data Availability Statement: All data generated are contained in the present manuscript.

Acknowledgments: G.D.M., H.M.-O., E.V. and D.M. are supported by the National Institute for Health Research (NIHR) Leeds Biomedical Research Centre (LBRC). The views expressed are those of the authors and not necessarily those of the (UK) National Health Service (NHS), the NIHR, or the (UK) Department of Health.

Conflicts of Interest: The authors declare no conflict of interest.

\section{References}

1. Bonaccorsi, G.; Pierri, F.; Cinelli, M.; Flori, A.; Galeazzi, A.; Porcelli, F.; Schmidt, A.L.; Valensise, C.M.; Scala, A.; Quattrociocchi, W.; et al. Economic and social consequences of human mobility restrictions under COVID-19. Proc. Natl. Acad. Sci. USA 2020, 117, 15530-15535. [CrossRef] [PubMed]

2. Neerukonda, S.N.; Katneni, U. A Review on SARS-CoV-2 Virology, Pathophysiology, Animal Models, and Anti-Viral Interventions. Pathogens 2020, 9, 426. [CrossRef] [PubMed]

3. Onyeaka, H.; Al-Sharify, Z.T.; Ghadhban, M.Y.; Al-Najjar, S.Z. A review on the advancements in the development of vaccines to combat coronavirus disease 2019. Clin. Exp. Vaccine Res. 2021, 10, 6-12. [CrossRef]

4. Golob, J.L.; Lugogo, N.; Lauring, A.S.; Lok, A.S. SARS-CoV-2 vaccines: A triumph of science and collaboration. JCI Insight 2021. [CrossRef]

5. Li, Y.D.; Chi, W.Y.; Su, J.H.; Ferrall, L.; Hung, C.F.; Wu, T.C. Coronavirus vaccine development: From SARS and MERS to COVID-19. J. Biomed. Sci. 2020, 27, 104. [CrossRef] [PubMed] 
6. Ball, P. The lightning-fast quest for COVID vaccines-And what it means for other diseases. Nature 2021, 589, 16-18. [CrossRef] [PubMed]

7. Polack, F.P.; Thomas, S.J.; Kitchin, N.; Absalon, J.; Gurtman, A.; Lockhart, S.; Perez, J.L.; Pérez Marc, G.; Moreira, E.D.; Zerbini, C.; et al. Safety and Efficacy of the BNT162b2 mRNA Covid-19 Vaccine. N. Engl. J. Med. 2020, 383, 2603-2615. [CrossRef] [PubMed]

8. Baden, L.R.; El Sahly, H.M.; Essink, B.; Kotloff, K.; Frey, S.; Novak, R.; Diemert, D.; Spector, S.A.; Rouphael, N.; Creech, C.B.; et al Efficacy and Safety of the mRNA-1273 SARS-CoV-2 Vaccine. N. Engl. J. Med. 2021, 384, 403-416. [CrossRef] [PubMed]

9. Voysey, M.; Clemens, S.A.C.; Madhi, S.A.; Weckx, L.Y.; Folegatti, P.M.; Aley, P.K.; Angus, B.; Baillie, V.L.; Barnabas, S.L.; Bhorat, Q.E.; et al. Safety and efficacy of the ChAdOx1 nCoV-19 vaccine (AZD1222) against SARS-CoV-2: An interim analysis of four randomised controlled trials in Brazil, South Africa, and the UK. Lancet 2021, 397, 99-111. [CrossRef]

10. Ramasamy, M.N.; Minassian, A.M.; Ewer, K.J.; Flaxman, A.L.; Folegatti, P.M.; Owens, D.R.; Voysey, M.; Aley, P.K.; Angus, B.; Babbage, G.; et al. Safety and immunogenicity of ChAdOx1 nCoV-19 vaccine administered in a prime-boost regimen in young and old adults (COV002): A single-blind, randomised, controlled, phase 2/3 trial. Lancet 2021, 396, 1979-1993. [CrossRef]

11. Ella, R.; Vadrevu, K.M.; Jogdand, H.; Prasad, S.; Reddy, S.; Sarangi, V.; Ganneru, B.; Sapkal, G.; Yadav, P.; Abraham, P.; et al. Safety and immunogenicity of an inactivated SARS-CoV-2 vaccine, BBV152: A double-blind, randomised, phase 1 trial. Lancet Infect. Dis. 2021. [CrossRef]

12. Zhang, Y.; Zeng, G.; Pan, H.; Li, C.; Hu, Y.; Chu, K.; Han, W.; Chen, Z.; Tang, R.; Yin, W.; et al. Safety, tolerability, and immunogenicity of an inactivated SARS-CoV-2 vaccine in healthy adults aged 18-59 years: A randomised, double-blind, placebocontrolled, phase 1/2 clinical trial. Lancet Infect. Dis. 2021, 21, 181-192.

13. Wise, J. Covid-19: Rare immune response may cause clots after AstraZeneca vaccine, say researchers. BMJ 2021, 373 , n954. [CrossRef]

14. Dotan, A.; Muller, S.; Kanduc, D.; David, P.; Halpert, G.; Shoenfeld, Y. The SARS-CoV-2 as an instrumental trigger of autoimmunity. Autoimmun. Rev. 2021, 20, 102792. [CrossRef]

15. Wucherpfennig, K.W. Mechanisms for the induction of autoimmunity by infectious agents. J. Clin. Invest. 2001, 108, 1097-1104. [CrossRef] [PubMed]

16. Torres-Aguilar, H.; Sosa-Luis, S.A.; Aguilar-Ruiz, S.R. Infections as triggers of flares in systemic autoimmune diseases: Novel innate immunity mechanisms. Curr. Opin. Rheumatol. 2019, 31, 525-531. [CrossRef] [PubMed]

17. Bragazzi, N.L.; Watad, A.; Sharif, K.; Adawi, M.; Aljadeff, G.; Amital, H.; Shoenfeld, Y. Advances in our understanding of immunisation and vaccines for patients with systemic lupus erythematosus. Expert Rev. Clin. Immunol. 2017, 13, 939-949. [CrossRef] [PubMed]

18. Toussirot, É.; Bereau, M. Vaccination and Induction of Autoimmune Diseases. Inflamm. Allergy Drug Targets 2015, 14, 94-98. [CrossRef]

19. Vera-Lastra, O.; Medina, G.; Cruz-Dominguez, M.D.P.; Jara, L.J.; Shoenfeld, Y. Autoimmune/inflammatory syndrome induced by adjuvants (Shoenfeld's syndrome): Clinical and immunological spectrum. Expert Rev. Clin. Immunol. 2013, 9, 361-373.

20. Pellegrino, P.; Clementi, E.; Radice, S. On vaccine's adjuvants and autoimmunity: Current evidence and future perspectives. Autoimmun. Rev. 2015, 14, 880-888. [CrossRef]

21. Gherardi, R.K.; Aouizerate, J.; Cadusseau, J.; Yara, S.; Authier, F.J. Aluminum adjuvants of vaccines injected into the muscle: Normal fate, pathology and associated disease. Morphologie 2016, 100, 85-94. [CrossRef]

22. Gherardi, R.K. Myofasciite à macrophages et hydroxyde d'aluminium: Vers la définition d'un syndrome des adjuvants [Lessons from macrophagic myofasciitis: Towards definition of a vaccine adjuvant-related syndrome]. Rev. Neurol. 2003, 159, 162-164. (In French)

23. Shoenfeld, Y.; Agmon-Levin, N. 'ASIA'—autoimmune/inflammatory syndrome induced by adjuvants. J. Autoimmun. 2011, 36, 4-8. [CrossRef]

24. Ameratunga, R.; Gillis, D.; Gold, M.; Linneberg, A.; Elwood, J.M. Evidence Refuting the Existence of Autoimmune/Autoinflammatory Syndrome Induced by Adjuvants (ASIA). J. Allergy Clin. Immunol. Pract. 2017, 5, 1551-1555.e1. [CrossRef]

25. Blasco, L.M. Refutation is a strong word for partial evidence in ASIA. J. Allergy Clin. Immunol. Pract. 2018, 6, 707-708. [CrossRef]

26. Tatematsu, M.; Funami, K.; Seya, T.; Matsumoto, M. Extracellular RNA Sensing by Pattern Recognition Receptors. J. Innate Immun. 2018, 10, 398-406. [CrossRef]

27. Teijaro, J.R.; Farber, D.L. COVID-19 vaccines: Modes of immune activation and future challenges. Nat. Rev. Immunol. 2021, 21, 195-197. [CrossRef]

28. Rodero, M.P.; Crow, Y.J. Type I interferon-mediated monogenic autoinflammation: The type I interferonopathies, a conceptual overview. J. Exp. Med. 2016, 213, 2527-2538. [CrossRef]

29. Krug, A. Nucleic acid recognition receptors in autoimmunity. Handb. Exp. Pharmacol. 2008, 129-151. [CrossRef]

30. El-Gabalawy, H.; Guenther, L.C.; Bernstein, C.N. Epidemiology of immune-mediated inflammatory diseases: Incidence, prevalence, natural history, and comorbidities. J. Rheumatol. Suppl. 2010, 85, 2-10. [CrossRef]

31. Chandler, R.E. Optimizing safety surveillance for COVID-19 vaccines. Nat. Rev. Immunol. 2020, 20, 451-452. [CrossRef] [PubMed]

32. Danova, J.; Kocourkova, A.; Celko, A.M. Active surveillance study of adverse events following immunisation of children in the Czech Republic. BMC Public Health 2017, 17, 167. [CrossRef] [PubMed] 
33. Gagnier, J.J.; Kienle, G.; Altman, D.G.; Moher, D.; Sox, H.; Riley, D.; CARE Group. The CARE guidelines: Consensus-based clinical case reporting guideline development. J. Med. Case Rep. 2013, 7, 223. [CrossRef] [PubMed]

34. World Health Organization. Global Manual on Surveillance of Adverse Events Following Immunization, 2016 Update. World Health Organization. 2014. Available online: https:/ /apps.who.int/iris/handle/10665/206144 (accessed on 27 April 2021).

35. Bellavite, P. Causality assessment of adverse events following immunization: The problem of multifactorial pathology. F1000Research 2020, 9, 170. [CrossRef]

36. Lee, H.; Kang, H.Y.; Cho, S.; Park, S.; Kim, A.Y.; Jung, S.Y.; Seong, B.L.; Lee, Y.M. Causality Assessment Guidelines for Adverse Events Following Immunization with a Focus on Guillain-Barré Syndrome. Vaccines 2020, 8, 101. [CrossRef]

37. Koenig, H.C.; Sutherland, A.; Izurieta, H.S.; McGonagle, D. Application of the immunological disease continuum to study autoimmune and other inflammatory events after vaccination. Vaccine 2011, 29, 913-919. [CrossRef]

38. McGonagle, D.; McDermott, M.F. A proposed classification of the immunological diseases. PLoS Med. 2006, 3, e297. [CrossRef]

39. López-González, M.D.; Peral-Garrido, M.L.; Calabuig, I.; Tovar-Sugrañes, E.; Jovani, V.; Bernabeu, P.; García-Sevila, R.; LeónRamírez, J.M.; Moreno-Perez, O.; Boix, V.; et al. Case series of acute arthritis during COVID-19 admission. Ann. Rheum. Dis. 2020. [CrossRef]

40. Segal, Y.; Shoenfeld, Y. Vaccine-induced autoimmunity: The role of molecular mimicry and immune cross-reaction. Cell. Mol. Immunol. 2018, 15, 586-594. [CrossRef]

41. Hussein, H.M.; Rahal, E.A. The role of viral infections in the development of autoimmune diseases. Crit. Rev. Microbiol. 2019, 45, 394-412. [CrossRef]

42. Lazarus, J.V.; Ratzan, S.C.; Palayew, A.; Gostin, L.O.; Larson, H.J.; Rabin, K.; Kimball, S.; El-Mohandes, A. A global survey of potential acceptance of a COVID-19 vaccine. Nat. Med. 2021, 27, 225-228. [CrossRef]

43. Duthie, M.S.; Windish, H.P.; Fox, C.B.; Reed, S.G. Use of defined TLR ligands as adjuvants within human vaccines. Immunol. Rev. 2011, 239, 178-196. [CrossRef]

44. McGonagle, D.; Bridgewood, C.; Ramanan, A.V.; Meaney, J.F.M.; Watad, A. COVID-19 vasculitis and novel vasculitis mimics. Lancet Rheumatol. 2021. [CrossRef]

45. Saad, M.A.; Alfishawy, M.; Nassar, M.; Mohamed, M.; Esene, I.N.; Elbendary, A. Covid-19 and Autoimmune Diseases: A Systematic Review of Reported Cases. Curr. Rheumatol. Rev. 2021, 33, 150.

46. Liu, Y.; Sawalha, A.H.; Lu, Q. COVID-19 and autoimmune diseases. Curr. Opin. Rheumatol. 2021, 33, $155-162$.

47. Ehrenfeld, M.; Tincani, A.; Andreoli, L.; Cattalini, M.; Greenbaum, A.; Kanduc, D.; Alijotas-Reig, J.; Zinserling, V.; Semenova, N.; Amital, H.; et al. Covid-19 and autoimmunity. Autoimmun. Rev. 2020, 19, 102597.

48. Castells, M.C.; Phillips, E.J. Maintaining Safety with SARS-CoV-2 Vaccines. N. Engl. J. Med. 2021, 384, 643-649. [CrossRef]

49. Benucci, M.; Infantino, M.; Marotto, D.; Ardizzone, S.; Manfredi, M.; Sarzi-Puttini, P. Vaccination against SARS-CoV-2 in patients with rheumatic diseases: Doubts and perspectives. Clin. Exp. Rheumatol. 2021, 39, 196-202.

50. Cirillo, N. Reported orofacial adverse effects of COVID-19 vaccines: The knowns and the unknowns. J. Oral Pathol. Med. 2021. [CrossRef]

51. Terracina, K.A.; Tan, F.K. Flare of rheumatoid arthritis after COVID-19 vaccination. Lancet Rheumatol. 2021. [CrossRef]

52. Blumenthal, K.G.; Robinson, L.B.; Camargo CAJr Shenoy, E.S.; Banerji, A.; Landman, A.B.; Wickner, P. Acute Allergic Reactions to mRNA COVID-19 Vaccines. JAMA 2021, e213976. [CrossRef]

53. Meo, S.A.; Bukhari, I.A.; Akram, J.; Meo, A.S.; Klonoff, D.C. COVID-19 vaccines: Comparison of biological, pharmacological characteristics and adverse effects of Pfizer/BioNTech and Moderna Vaccines. Eur. Rev. Med. Pharmacol. Sci. 2021, 25, 1663-1669. [CrossRef]

54. Klimek, L.; Eckrich, J.; Hagemann, J.; Casper, I.; Huppertz, J. Allergische Reaktionen auf COVID-19-Impfstoffe-Evidenz und praxisorientiertes Vorgehen [Allergic reactions to COVID-19 vaccines: Evidence and practice-oriented approach]. Internist 2021, 62, 326-332. (In German) [CrossRef]

55. CDC COVID-19 Response Team; Food and Drug Administration. Allergic Reactions Including Anaphylaxis After Receipt of the First Dose of Pfizer-BioNTech COVID-19 Vaccine-United States, December 14-23, 2020. MMWR Morb. Mortal. Wkly. Rep. 2021, 70, 46-51. [CrossRef] 